\title{
Über die physikalische Bedeutung der klassischen elektrodynamischen Potentiale
}

\author{
von \\ Jörg Pfleiderer
}

(vorgelegt in der Sitzung der math.-nat. Klasse am 14. Oktober 2010 durch das w. M. Pfleiderer Jörg)

\section{Zusammenfassung}

Im Sinne des klassischen Dualismus elektrischer Ladungen als Teilchen und Feld wird das auf eine mit der Geschwindigkeit $\boldsymbol{v}$ bewegte Ladung $e$ wirkende skalare Potential $\boldsymbol{e U}$, das Vektorpotential $\boldsymbol{e A}$, das Vektorprodukt $\boldsymbol{r} \times e \boldsymbol{A}$ und das Skalarprodukt $\boldsymbol{e v \boldsymbol { A }}$ interpretiert als jeweils integrale Wechselwirkungsgröße des Coulomb-Felds oder des Magnetfelds der Ladung mit äußeren Feldern, sprich elektrische Feldenergie, Feldimpuls, Felddrehimpuls und magnetische Feldenergie. Globale Coulomb-Eichung oder die Beschränkung auf den global eichinvarianten quellenfreien (transversalen) Teil von $\boldsymbol{A}$ muß hier angenommen werden. Dazu passend wird (als Näherung zweiter Ordnung in $v / c$, entsprechend einer Vernachlässigung der Strahlungsdämpfung) eine jeweils nicht explizit zeitabhängige Lagrange- und Hamilton-Funktion aufgestellt, aus denen die üblichen 7 skalaren Erhaltungsgrößen in einem abgeschlossenen System (Energie, Impuls, Drehimpuls) ableitbar sind. Zwischen euphysikalischen (eine externe physikalische Situation beschreibenden) und aphysikalischen (auf einer mathematischen Identität beruhenden) Eichtransformationen kann zwar global, aber nicht lokal unterschieden werden.

Vorbemerkung: Es war ein früheres Mitglied unserer Akademie, Erwin Schrödinger, der mich ermutigt hat, die folgenden Gedanken ohne Rücksicht auf die Meinung von Kollegen weiter zu verfolgen.

\section{Einleitung}

Ein klassischer (Newtonscher) Gravitationsmassenpunkt ist eine verhältnismäßig einfache Angelegenheit. Er ist durch zwei Angaben 
bestimmt: Ort und Masse. Um genauer zu sein, hat er eine Ausdehnung, die genügend klein ist, um ihn nicht wesentlich von einem ausdehnungslosen Punkt zu unterscheiden. Seine Bewegung läßt sich durch 3 kinetische (7 skalare) Bestimmungsgrößen charakterisieren: Energie, Impuls und Drehimpuls. Seine Wechselwirkung mit anderen Massen erfolgt über eine Fernwirkungskraft, die Gravitation. Diese, d.h. die auf den Massenpunkt wirkende Kraft, wird beschrieben durch den Gradienten eines skalaren Potentials, entsprechend einer potentiellen Energie oder gravitativen Wechselwirkungsenergie. Bei der Wechselwirkung zwischen Massenpunkten werden Impuls und Drehimpuls insgesamt erhalten, während Energie zwischen kinetischer und potentieller Energie ausgetauscht wird, unter Beibehaltung der Summe beider, d.h. unter Erhaltung der Gesamtenergie.

Das Potential wird nicht direkt gemessen, sondern nur über die daraus resultierende Kraft, d.h. über den Gradienten des Potentials. Dementsprechend kann man eine skalare Funktion mit verschwindendem Gradienten, d.h. eine Konstante, hinzufügen, ohne das physikalische Problem, nämlich die Wechselwirkung, zu beeinflussen. Die Energieerhaltung läßt es ebenso zu, der Bilanz eine Konstante hinzuzufügen. Allgemeiner darf die Konstante sogar zeithängig sein. Demnach bedarf es einer mehr oder weniger beliebigen Übereinkunft (Eichung) bezüglich dieser Konstanten, um dem skalaren Potential und der potentiellen Energie bestimmte Werte zuzuordnen. Während das Potential der Eichung unterliegt, ist der Gradient davon unabhängig, d.h. eichinvariant. In der Himmelsmechanik ist es üblich, die Eichung so zu wählen, daß das Potential im Unendlichen verschwindet. Eine Masse im Unendlichen hat dann nicht nur keine Wechselwirkung mit einer Masse im Endlichen (diese Aussage ist eichinvariant), sondern erzeugt auch keine potentielle Wechselwirkungsenergie (diese Aussage ist nicht eichinvariant).

Nur diese Eichung lässt eine direkte physikalische Interpretation zu: Dann entspricht nämlich die potentielle Energie gerade dem (eindeutigen) Aufwand, der zur Heranführung der Masse aus dem Unendlichen ceteris paribus nötig wäre bzw. frei würde.

Ein bekanntes und keineswegs nur in der Himmelsmechanik benütztes Beispiel dieser Eichung ist das Virialtheorem: Im Gleichgewichtszustand eines Systems von Massenpunkten (als himmelsmechanisches Beispiel: Ein Sternhaufen) ist die - negative - potentielle Energie $V$ doppelt so groß wie die kinetische Energie $T$, d.h. $2 T+V=0$.

Trotzdem ist - oder jedenfalls erscheint - diese Eichung, von einem rein formalen Standpunkt aus, willkürlich, obwohl sie zweifellos physikalisch sinnvoll ist. 
In der Elektrodynamik ist alles ein wenig komplizierter. Zwar hat eine klassische Punktladung $e$ eine Masse $m$; ihre Bewegung $v$ ist wie im Gravitationsfall durch kinetische Bestimmungsgrößen (Energie, Impuls, Drehimpuls) charakterisiert. Sie unterliegt aber zweierlei Kräften. Die eine, elektrische, ist durch ein skalares Potential $U$ nur zum Teil definiert, die andere, magnetische, bedarf sogar eines neuen Konzepts, des Vektorpotentials $\boldsymbol{A}$. Wichtiger ist, daß die Ladung außerdem elektromagnetische Felder um sich erzeugt oder hat, welche - was gelegentlich übersehen wird - als essentieller Bestandteil der Ladung zu gelten haben, nicht nur als rechnerisches Hilfsmittel, um die Wirkung auf andere Ladungsträger zu beschreiben. Diese Felder sind ausgedehnt. Sie sind ebenfalls durch die genannten Bestimmungsgrößen charakterisiert, lokal als Felddichten, gesamt als Dichte-Integrale. Diese Felddichten sind ausgedehnt und nicht per se auf einen Punkt lokalisiert. Die Symmetrie der Feldverteilung sorgt allerdings dafür, daß die gemittelte Feldposition mit dem Ort der Ladung übereinstimmt.

$\mathrm{Da} ß$ die Felder kein unwesentliches Beiwerk der Ladungen sind, mag aus der Definition des klassischen Elektronenradius erhellen: Die gesamte Massenenergie des Elektrons ist in die Feldenergie des umgebenden elektrischen Coulomb-Felds gepackt. Ebenso wird die kinetische Energie des bewegten klassischen Elektrons mit diesem Radius in die magnetische Feldenergie gepackt, der kinetische Impuls in den Feldimpuls. Für andere Ladungen sind freilich diese Feldgrößen immer klein gegenüber Ruheenergie, kinetischer Energie und kinetischem Impuls, im Großen und Ganzen um mindestens den Faktor $m_{e} / m_{\mathrm{p}}$ bzw. einer Potenz davon. Dennoch ist immer ein Teil der Ruhemasse einer Ladung, auch einer makroskopischen, elektromagnetischen Ursprungs.

Ein klassisches Elektron ist ebenso wie jede andere klassische Ladung sowohl ein Punkt (oder Fast-Punkt) als Ladung als auch unendlich ausgedehnt als Feld. Dieser klassische Dualismus kommt meiner Meinung nach in der Diskussion deutlich zu kurz.

Die Bewegung einer Ladung bzw. die Lorentz-Kraft auf die Ladung ist durch zwei Felder, elektrisches und magnetisches, bestimmt. Es ist nicht nötig, sich mit der Frage zu beschäftigen, ob die Kräfte auf die Ladung oder auf die dazugehörigen Felder wirken, denn es genügt wegen der Symmetrie der Feldverteilung, die Kräfte am Ort der Ladung zu kennen. Die mit der Ladung verbundenen Felder bewegen sich sozusagen von selbst mit der Ladung mit. Ich erwähne nur nebenbei, daß man dies selbstverständlich im einzelnen nachvollziehen kann. Die Felder können, so scheint es also zunächst, erst dann ein physikalisches 
Eigenleben entwickeln, wenn sie sich von den Ladungen trennen, d.h. in Form von Strahlung - allgemeiner in Form von elektromagnetischen Wellen - auftreten.

Elektromagnetische Felder sind, ebenso wie die sie beschreibenden Potentiale, linear superponierbar - eine Folge der Linearität der Maxwellschen Gleichungen. Das impliziert, daß sich die Felder von zwei Ladungen überlagern und dabei eine Überlagerungs-Feldenergiedichte oder Wechselwirkungs-Feldenergiedichte erzeugen, deren mittlere Position nicht mit der Position einer der Ladungen übereinstimmt. Diese Überlagerungsenergie bedarf daher einer besonderen Behandlung. Dasselbe gilt für den Überlagerungs-Feldimpuls bzw. -Felddrehimpuls.

Die Überlagerungsgrößen zweier Ladungen sind äußerst klein gegenüber den Einzelgrößen, nämlich ungefähr um eine Potenz des Faktors $r_{e} / r=$ klassischer Elektronenradius/Abstand der Ladungen. Bei einem Vielteilchenproblem mit $\mathrm{N}$ Teilchen können aber die Einzelgrößen nur mit $\mathrm{N}$, die Überlagerungsgrößen jedoch mit $\mathrm{N}^{2}$ wachsen. Alle makrophysikalischen elektromagnetischen Effekte sind Überlagerungseffekte. Deshalb spielen dort, z.B. in der Plasmaphysik, die Feldgrößen eine wichtige Rolle, während sie in der Zweiteilchenphysik als Störungen behandelt werden können. Jede Theorie, die äußere Einflüsse kennt, z.B. die Bewegung einer Ladung unter äußeren Feldern, ist eine Vielteilchentheorie. Daher dürfen kleine Wechselwirkungseffekte in der Formulierung nicht unterschätzt werden.

Bekanntlich sind die Potentiale nicht eindeutig bestimmt. Ohne die Kräfte auf die Ladungen, also das elektrische und das magnetische Feld, zu verändern, kann man zum Vektorpotential den Gradienten einer beliebigen Funktion von Ort und Zeit $f(\boldsymbol{r}, t)$ hinzufügen, wenn das skalare Potential gleichzeitig durch die Zeitableitung von $f$ ergänzt wird. Das nennt man Eichtransformation. Anders gesagt: Die Bewegungsgleichungen der Ladungen sind unter diesen Transformationen eichinvariant. Die physikalischen Resultate, nämlich wie sich die Ladungen bewegen, werden durch eine Eichtransformation nicht modifiziert. Das führt zu einer Lehrbuch-Aussage etwa der folgenden Form (z.B. Cohen-Tannoudji et al. 2009=,„CT1“): Das skalare und das Vektorpotential treten nur als Zwischengrößen bei der Berechnung der elektrischen und der magnetischen Feldstärke auf. Sie haben in der klassischen Physik keine physikalische Relevanz. Die wichtigen Größen in der Elektrodynamik sind allein die Felder.

Es sind die (von mir) kursiv und fett geschriebenen Wörter, die ersatzlos gestrichen werden sollten. 
Solche Lehrbuch-Aussagen sind freilich keineswegs neueren Datums. Beispielsweise erwähnt v. Laue (1956) eine Erweiterung der Elektrodynamik durch Mie (1912), bei der unter anderem ,das Viererpotential, welches bisher lediglich ein mathematisches Hilfsmittel war, eine physikalische Bedeutung bekommt".

Mit den ,wichtigen Größen“ sind offenbar die Felder gemeint, welche die Bewegung einer Ladung bestimmen, während von den von einer Ladung erzeugten Feldern nicht die Rede ist, außer insofern als sie wiederum die Bewegung anderer Ladungen beeinflussen. Kurz, über Feldenergie und Feldimpuls wird oft genug nichts gesagt. Diese Größen kennt man eigentlich fast nur aus der Makrophysik, bei der Überlagerungsfelder als Energie- und Impulsträger eine viel größere Rolle spielen, z.B. aus der Plasmaphysik oder noch einfacher aus der Elektrostatik, und selbst da ist ihre Bedeutung, ja sogar ihre Gültigkeit keineswegs immer klar gewesen.

CT1 unterscheidet zwischen wahren physikalischen Größen und nichtphysikalischen Größen. Die Werte der ersten Art sind durch das physikalische Problem festgelegt - z.B. gibt das elektrische Feld die Kraft auf eine Ladung. Diese Größen sind eichinvariant und können direkt gemessen werden. Größen der zweiten Art können nicht direkt gemessen werden. An einem gegebenen Ort zu gegebener Zeit können sie, je nach Eichung, jeden beliebigen Wert annehmen, ohne die dazugehörige Physik zu ändern.

Das Konzept zweier Arten von physikalischen Größen führt zu merkwürdigen Konsequenzen. Die Theorie erfordert, wahre und nichtphysikalische Größen durcheinander zu mischen. Als Beispiel ist der kanonische Impuls die Summe einer wahren (kinetischer Impuls) und einer nicht- oder unphysikalischen (Vektorpotential) Größe, womit der kanonische Impuls als Ganzes in der klassischen Physik ebenfalls zu einer nichtphysikalischen Größe wird. Das allein ist schon erstaunlich genug. Aber in der Quantenphysik wird der kanonische Impuls trotz Eichabhängigkeit als zwar nichtphysikalische, aber dennoch als wesentliche physikalische Größe angesehen, wodurch der kinetische Impuls zu einer nichtphysikalischen oder doch sekundären, unwesentlichen wird, und zwar hauptsächlich aus formalen Gründen. Im rein mechanischen Fall ist der kinetische Impuls nämlich auch in der Quantenphysik eine wahre physikalische Größe.

Ich halte die Bezeichnung „nichtphysikalisch“ oder - fast noch schlimmer - ,unphysikalisch“ für reichlich irreführend. Bei weitem besser, nämlich klarstellend, ist die Beschreibung durch Grübl (2010): „Diese Größen enthalten neben physikalischen Fakten auch willkürliche Eichkonvention“. Damit ist alles Wichtige gesagt. 
Die zum großen Teil tatsächlich längst bekannten physikalischen Fakten in den Potentialen und einigen davon abhängigen Funktionen aufzuzeigen, ist die Intention der vorliegenden Arbeit. Damit wird keine neue Theorie aufgestellt, sondern nur Lehrbuchwissen anders interpretiert. Deshalb wäre auch eine ausführlichere Literaturzitation von wenig Wert.

Im Rahmen einer quasistationären Näherung werden Strahlungsfelder, die nicht an Ladungen gekoppelt sind, vernachlässigt. Das muß ich auch hier tun. Wie man z.B. bei Landau \& Lifschiz (1963, $=$,LL63“) nachlesen kann, ist hiervon insbesondere die explizite, d.h. nicht explizit zeitabhängige Schreibweise der Potentiale betroffen.

\section{Interpretation der Potentiale}

Zunächst einige Fragen, die in vielen Lehrbüchern nicht beantwortet werden:

Eine Ladung, die unter dem Einfluß von (äußeren) Feldern steht, erzeugt notwendig Wechselwirkungs-Feldimpuls und Wechselwirkungs-Feldenergien. Diese müssen ebenso notwendigerweise irgendwo in den Erhaltungsgleichungen auftauchen. Aber wo?

Da man die Felder, auch die Überlagerungsfelder, lokal durch Felddichten beschreibt, wird man nach Integralgrößen suchen müssen. Aber wie?

Umgekehrt tauchen die Potentiale (mit geeigneten Faktoren) zum Beispiel in der Lagrange-Funktion mit Dimensionen von Impuls und Energie auf. Was für Impulse bzw. Energien könnten das sein?

In mechanischen Problemen enthält die Hamilton-Funktion nicht nur die Bewegungsgleichung bzw. -gleichungen, sondern auch Aussagen über Erhaltungsgrößen, nämlich über Energie, kanonischen Impuls und kanonischen Drehimpuls. Wie sieht das im elektrodynamischen Fall aus?

Da die lokalen Felder durch Ableitungen der Potentiale bestimmt sind, sind die Potentiale selbst als eine Art Integralgrößen anzusehen. Hier nach einer Verbindung zu suchen, liegt meines Erachtens recht nahe. Integralgrößen haben gewöhnlich eine prinzipielle Unbestimmtheit, soweit die Integrationsgrenzen nicht eindeutig sind. Gibt es einen Zusammenhang mit dem Eichproblem?

Im folgenden werden die Terme $e U, e \boldsymbol{A}, \boldsymbol{r} \times e \boldsymbol{A}$ und $e \boldsymbol{v} \boldsymbol{A}$ gedeutet, genauer gesagt der jeweils eichinvariante Teil davon. Diese Interpretationen sind keineswegs neu (siehe z.B. Pfleiderer 1966, =,,P66“), aber erstaunlicherweise nahezu unbekannt. Wegen der Eichproblema- 
tik wird oft sogar auch heute noch, ebenso erstaunlicherweise, die bloße Möglichkeit einer solchen Interpretation abgelehnt.

\subsection{Skalares Potential}

Das folgende ist zwar meines Wissens altbekannt, aber ich kann keine Literaturstelle dazu angeben (außer P66). Angesichts der viel jüngeren Aussage von CT1, daß auch das skalare Potential eine nicht-physikalische Größe sei, d.h. eine mathematische Hilfsgröße ohne jede eigene physikalische Bedeutung, mag jedoch eine Neudarstellung sinnvoll sein. Ich möchte daran erinnern, daß eine (negative) GravitationsWechselwirkungs-Feldenergie im Rahmen der Newtonschen Gravitationstheorie ein zwar nicht notwendiges und auch nicht allgemein anerkanntes, aber durchaus sinnvolles Konzept darstellt.

Die potentielle Energie einer Ladung $e$ am Ort $\boldsymbol{r}$ in einem skalaren, durch das Potential $U$ beschriebenen elektrischen Feld sei $e U$. Dabei ist $U$ jedenfalls vom Ort $\boldsymbol{r}$ abhängig und beschreibt über den Gradienten am Ort $\boldsymbol{r}$ ein auf die Ladung wirkendes elektrisches Skalarfeld, das man gewöhnlich Coulomb-Feld oder elektrostatisches Feld nennt. Dieses Feld ist als Gradientenfeld einerseits wirbelfrei, andererseits notwendigerweise nicht überall quellenfrei. Die einzigen „euphysikalischen“ Quellen sind dabei irgendwo im Raum verteilte andere Ladungen.

Die Ladung $e$ läßt sich als Integral über die Raumladungsdichte $\rho$ schreiben:

$$
e=\int \rho d V=\int \operatorname{div} \boldsymbol{E} d V .
$$

Unter der Konvention, daß nur diese Ladung zur Raumladungsdichte in dem Integral beitrage, kann das Integral über den unendlichen Raum erstreckt werden (ebenso wie alle folgenden Integrale). Die Position $\underline{r}$ der Ladung ist durch

$$
\underline{r}=\frac{1}{e} \int \rho \boldsymbol{r} d V
$$

beschreibbar. Für das der klassischen Vorstellung zunächst entgegenkommende Konzept einer Punktladung wird ein solches Integral elegant durch eine Dirac'sche Deltafunktion formuliert. Ich vermeide dies hier, weil einerseits ausgedehnte Ladungen (Atome, Moleküle, selbst Molekülverbände und sogar viel Größeres) oft am besten durch Punktdarstellungen repräsentiert werden, und andererseits auch die 
kleinste klassische Ladung notwendig ausgedehnt sein muß (klassischer Elektronenradius). Dazu ist insbesondere auf Rohrlich (1964) hinzuweisen, der - nach meiner Kenntnis als erster - eine ebenso konsequente wie elegante klassische Formulierung entwickelte.

Für das Potential, das auf die Ladung wirkt, gilt entsprechend

$$
\underline{U}=\frac{1}{e} \int \rho U d V \text {. }
$$

In die Formeln geht hingegen das Potential $U(\underline{r})$ am , Ladungsort “ $\boldsymbol{r}$ ein. Anstatt durch eine Diracsche Deltafunktion die Gleichheit $\underline{U}=\overline{U(\underline{\boldsymbol{r}}) \mathrm{zu}}$ erzwingen, will ich hier nur - als schwächere Forderung - annehmen, daß die Differenz vernachlässigbar ist. Entsprechend soll auch der Unterschied zwischen $\nabla(\underline{U})$ und $(\nabla U)$ vernachlässigt werden.

Dann wird unter Weglassung aller Maßsystemskonstanten (oberer Index $i$ : intern, erzeugt durch Ladung $i$; oberer Index e: extern, wirkend auf die Ladung $i=$ von außen erzeugt)

$$
\begin{aligned}
e U & =\int \rho U d V=\int U^{e} \operatorname{div} \boldsymbol{E}^{i} d V=\int \operatorname{div}\left(U^{e} \boldsymbol{E}^{i}\right) d V-\int \boldsymbol{E}^{i} \operatorname{grad} U^{e} d V \\
& =0+\int \boldsymbol{E}^{i}\left(E^{e}+\frac{\partial A^{e}}{\partial t}\right) d V .
\end{aligned}
$$

Hier ist davon Gebrauch gemacht, daß das Raumintegral über die Ableitung (hier div) einer Funktion (hier $U^{e} \boldsymbol{E}^{i}$ ) nach dem Gaußschen Satz in ein Oberflächenintegral über diese Funktion umformbar ist. Dieses verschwindet im Grenzübergang gegen Unendlich, wenn die Funktion im Unendlichen wie mindestens $r^{-3}$ (genauer $r^{-2-\alpha}, \alpha>0$ ) verschwindet. Damit kann die Ableitung im Raumintegral unter Vorzeichenwechsel von einer Größe (Feld $\boldsymbol{E}$ der Ladung, das mit $r^{-2}$ abfällt) auf die andere Größe (hier Potential $U$, das mit jeder erzeugenden Ladung wie $r^{-1}$ abfällt, also auch insgesamt mindestens so, wenn sich alle Ladungen im Endlichen befinden) verschoben werden.

Das aus $\rho$ und $\operatorname{div} \boldsymbol{E}$ folgende elektrische Feld $\boldsymbol{E}^{i}$ (durch die Ladung $i$ erzeugtes Feld) ist das Coulombfeld der Ladung. Zunächst ist das durch Bewegung der Ladung erzeugbare elektrische Wirbelfeld oder Induktionsfeld der Ladung nicht ausgeschlossen, $\boldsymbol{E}^{i}$ könnte also durchaus das gesamte durch die Ladung erzeugte Feld - ohne Strahlung - sein. Das durch $\operatorname{grad} U$ beschriebene auf die Ladung $e$ wirkende Feld $\boldsymbol{E}^{e}+\partial \boldsymbol{A}^{e} / \partial t$ ist dagegen wirbelfrei. Das Wirbelfeld der Ladung trägt also zum Integral nichts bei. Das durch $\partial \boldsymbol{A} / \partial t$ beschriebene als Ergänzung zu grad $U$ gehörige äußere Wirbelfeld ist freilich nur dann ein reines 
Wirbelfeld, enthält also keine zum Integral beitragenden Gradientenanteile, wenn auch $\boldsymbol{A}$ keine solchen Anteile (genauer keine zeitabhängigen solchen Anteile) enthält. Das entspricht der Annahme

$$
\operatorname{div} \boldsymbol{A}=0
$$

(Coulomb-Eichung). Diese Eichung ist eine globale Forderung. Ihre Gültigkeit in einem beschränkten Gebiet - entsprechend einer Kenntnis der physikalischen Umstände nur in einem Teilgebiet - ist nicht ausreichend. Sie wäre nicht eindeutig.

In der Coulomb-Eichung - und zwar nur in dieser - gilt also: Die potentielle Energie $e U$ ist gleich der elektrischen Wechselwirkungsenergie $\int \boldsymbol{E}^{i} \boldsymbol{E}^{e} d V$, die durch die Überlagerung des Coulombfeldes der Ladung und des äußeren Coulombfeldes entsteht. Anders ausgedrückt: In der Beschreibung von Grübl (2010) besteht der Ausdruck $e U$ also aus der Wechselwirkungsenergie (physikalisches Faktum) plus einem - im allgemeinen nicht bekannten - Eichkonventionsanteil.

Die gesamte elektrische Feldenergie ${ }^{1} / 2 \int \boldsymbol{E}^{2} d V$ besteht aus einem Wirbelteil, der hier nicht aufscheint, und einem Quellenteil oder Coulombteil. Dieser letztere enthält drei Teile: Die Coulomb-Energie des äußeren Feldes $1 / 2 \int \boldsymbol{E}^{e 2} d V$, die (konstante) Eigenfeldenergie der Ladung $1 / 2 \int \boldsymbol{E}^{i 2} d V$, und eben die obige Wechselwirkungsenergie. Deshalb ist es, allerdings nur bei konstantem äußerem Feld, äquivalent, wenn man $e U$ bis auf eine Konstante mit der gesamten, nicht aufgeteilten elektrischen Coulomb-Energie $1 / 2 \int \boldsymbol{E}_{\mathrm{C}}{ }^{2}$ $d V$ identifiziert.

Das Superpositionsprinzip - eine Folge der Linearität der Maxwell'schen Gleichungen - besagt, daß man Felder und Potentiale addieren kann. Entsprechend ist die Wechselwirkung der Ladung $i$ mit anderen Ladungen $j$ (d.h. $j \neq i$ ) und mit im strengeren Sinne äußeren Bedingungen e (extern) beschreibbar durch das Potential

$$
U_{i}=\sum_{j \neq i} U_{i j}+U_{i e}
$$

und die Wechselwirkungsenergie durch eine Summe über die Wechselwirkung mit anderen Ladungen $j$ und dem im strengeren Sinne äußeren (Coulomb-)Feld.

Die Wechselwirkungspotentiale $U_{i j}$ sind - in einer Näherung zweiter Ordnung in $v / c$ - durch den Ort beider Partner, genauer durch die Ortsdifferenz bestimmt, lassen sich also explizit zeitunabhängig (d.h. genauer: nicht explizit zeitabhängig) als Funktion von $r_{i j}=\left|\boldsymbol{r}_{i}-\boldsymbol{r}_{j}\right|$ beschreiben. Das auf ,echt äußere“ Felder zurückführbare äußere 
Potential $U_{i e}$ ist bekanntlich als eine Funktion des Ortes $\boldsymbol{r}_{i}$ der Ladung $e_{i}$ und möglicherweise der Zeit zu geben. Diese Zeitabhängigkeit ist nicht reduzierbar, solange man die äußeren Prozesse nicht im einzelnen beschreibt. Insbesondere pflegt man die Rückwirkung des Testkörpers (hier der Ladung) auf das äußere System zu vernachlässigen und hat deshalb die Gültigkeit bzw. Ungültigkeit von Erhaltungssätzen im einzelnen zu prüfen (bekanntes Beispiel: Keine Impulserhaltung im Einkörperproblem).

\subsection{Vektorpotential}

Neben den oberen Indizes ${ }^{i}$ und ${ }^{e}$ am Anfang und am Ende benütze ich hier die unteren Indizes 1 und 2 als Zeichen, auf welchen der Integrandenbestandteile die Ortsableitung $\nabla \mathrm{zu}$ wirken hat. Eine ähnliche Rechnung wie oben, jedoch mit dem Vektorpotential, führt dann auf

$$
\begin{aligned}
e \boldsymbol{A}= & \int \rho \boldsymbol{A} d V=\int \boldsymbol{A}^{e} \operatorname{div} \boldsymbol{E}^{i} d V=\int \boldsymbol{A}_{2}\left(\nabla_{1} \boldsymbol{E}_{1}\right) d V \\
= & \int\left(\boldsymbol{E}_{1} \times\left(\boldsymbol{A}_{2} \times \nabla_{1}\right)+\nabla_{1}\left(\boldsymbol{E}_{1} \boldsymbol{A}_{2}\right)\right) d V \\
= & \int\left(\boldsymbol{E}_{1} \times\left(\boldsymbol{A}_{2} \times \nabla_{1,2}\right)+\nabla_{1,2}\left(\boldsymbol{E}_{1} \boldsymbol{A}_{2}\right)\right) d V+\int \boldsymbol{E}_{1} \times\left(\nabla_{2} \times \boldsymbol{A}_{2}\right) d V \\
& -\int \nabla_{2}\left(\boldsymbol{E}_{1} \boldsymbol{A}_{2}\right) d V=0+\int \boldsymbol{E}^{i} \times \boldsymbol{B}^{e} d V-\int \nabla_{2}\left(\boldsymbol{E}_{1} \boldsymbol{A}_{2}\right) d V
\end{aligned}
$$

mit

$$
\begin{aligned}
\int \nabla_{2}\left(\boldsymbol{E}_{1} \boldsymbol{A}_{2}\right) d V & =\int\left\{\boldsymbol{A}_{2} \times\left(\nabla_{2} \times \boldsymbol{E}_{1}\right)-\boldsymbol{E}_{1} \operatorname{div} \boldsymbol{A}_{2}\right\} d V \\
& =-\int \boldsymbol{A}^{e} \times \operatorname{rot} \boldsymbol{E}^{i} d V-\int \boldsymbol{E}^{i} \operatorname{div} \boldsymbol{A}^{e} d V
\end{aligned}
$$

Das zweite Integral verschwindet in der Coulomb-Eichung, das erste dann, wenn man unter $\boldsymbol{E}$ das Coulomb-Feld der Ladung $e$ versteht. Die Integrale mit $\nabla_{1,2}$ verschwinden wie oben, da auch $\boldsymbol{A}$ mit $r^{-1}$ abfällt, wenn sich alle Ladungen im Endlichen befinden.

Schreibt man noch, im Sinne der Superposition,

$$
\boldsymbol{A}_{i}=\sum_{j \neq i} \boldsymbol{A}_{i j}+\boldsymbol{A}_{i e}
$$


so gilt in der Coulomb-Eichung, und nur in dieser, daß $e_{i} \boldsymbol{A}_{i}$ den Feldimpuls (das Integral über die Feldimpulsdichte $\int \boldsymbol{E} \times \boldsymbol{B} d V$ ) darstellt, der aus dem Coulombfeld der Ladung $i$ und dem durch die Bewegung der einzelnen anderen Ladungen $j$ jeweils entstandenen Magnetfeld bzw. dem äußeren Magnetfeld resultiert. Eine andere Formulierung desselben Tatbestands ist, daß hier nur der eichinvariante wirbelbehaftete und quellenfreie - der transversale - Teil $\boldsymbol{A}_{\perp}$ des Vektorpotentials einzusetzen ist.

Die Idee, das Vektorpotential mit einem Feldimpuls (= Feldimpulsdichte-Integral) pro Ladungseinheit zu identifizieren, ist nicht neu, aber nach meiner Kenntnis nie im Sinne einer klassischen Deutung näher verfolgt worden. Die älteste Literaturstelle, die ich kenne, ist eine Fußnote in einem amerikanischen Lehrbuch der Elektrodynamik aus den frühen 1960er Jahren, in der diese Gleichheit, allerdings sehr rigoros eingeschränkt auf einen eindimensionalen Sonderfall, als Besonderheit erwähnt wird. Trammel (1964) postuliert (meiner Kenntnis nach als erster) die Gleichheit, ohne sie allerdings vollständig zu beweisen. Seine durchaus überzeugende Formulierung ebenso wie mein ergänzender Beweis (P66) haben zu keiner mir bekannt gewordenen Reaktion geführt. Ungeachtet der oben zitierten klaren Aussage von CT1 über die klassische Nichtrelevanz der Potentiale ist jedoch genau diese Identifikation in Cohen-Tannoudji et al. 1997 (= „CT2“) ebenso unmißverständlich als klassische Gleichheit enthalten, sogar mit dem ausdrücklichen Zusatz, daß die Aussage eichinvariant ist, wenn man unter $\boldsymbol{A}$ den quellenfreien oder transversalen Anteil $\boldsymbol{A}_{\perp}$ versteht.

Das Vektorpotential in Coulomb-Eichung (das ist das Grübl'sche (l.c.) ,physikalische Faktum“) ist hiernach derjenige Impuls, den eine ruhende Einheitsladung zum Gesamtimpuls eines physikalischen Systems beiträgt. Die bewegte Ladung fügt dem zwei weitere Teile hinzu: Den kinetischen Impuls und den Feldimpuls, der aus dem äußeren elektrischen Feld und dem von der bewegten Ladung erzeugten Magnetfeld entsteht. Dieser Feldimpuls wird durch dasjenige Vektorpotential beschrieben, welches die bewegte Ladung am Ort anderer Ladungen erzeugt. Er wird sozusagen ebenso einer anderen Ladung zugeschlagen, wie deren Magnetfeld bzw. Vektorpotential durch den Ausdruck $\boldsymbol{e A}$ der betrachteten Ladung zugeschlagen wird.

\subsection{Felddrehimpuls}

Hier besteht die Schwierigkeit, daß die Impulsdichte mit einem Hebel (Abstand vom gewählten Ursprung) versehen wird, der die Verhältnisse 
im Übergang zum Unendlichen verändert. Die Feldimpulsdichte muß deshalb mindestens mit $r^{-3-\alpha}$ ins Unendliche abfallen, damit die auch hier auftretenden Oberflächenintegrale verschwinden. Das für die Probeladung belangreiche Vektorpotential muß entsprechend mit $r^{-1-\alpha}$ abfallen, was das Vektorpotential einer einzelnen Ladung nicht tut - genauer gesagt, in einer quasistatischen Näherung nicht tut. Es genügt aber, anzunehmen, daß die Gesamtladung des Systems verschwindet, d.h. daß die Beiträge positiver und negativer Ladungen sich hinreichend aufheben. Obwohl dies eine in allen vorstellbaren praktischen Fällen hinreichend erfüllte Bedingung ist, kann die Allgemeingültigkeit nur behauptet werden. Ich verweise deshalb auf die moderne Kosmologie, die davon ausgeht, daß das Universum insgesamt ungeladen ist. Die Einzelrechnung lautet

$$
\begin{aligned}
\boldsymbol{r} \times e \boldsymbol{A}= & \int \boldsymbol{r} \times \rho \boldsymbol{A} d V=\int \boldsymbol{r} \times \boldsymbol{A}^{e} \operatorname{div} \boldsymbol{E}^{i} d V=\int\left(\boldsymbol{r}_{2} \times \boldsymbol{A}_{3}\right)\left(\nabla_{1} \bullet \boldsymbol{E}_{1}\right) d V \\
= & \int\left\{\nabla_{1} \times\left(\left(r_{2} \times \boldsymbol{A}_{3}\right) \times \boldsymbol{E}_{1}\right)+\left(\left(r_{2} \times \boldsymbol{A}_{3}\right) \bullet \nabla_{1}\right) \boldsymbol{E}_{1}\right\} d V \\
= & \int \boldsymbol{r} \times\left(\boldsymbol{E}^{i} \times \boldsymbol{B}^{e}\right) d V \\
& +\int\{(\nabla(\boldsymbol{r} \times \boldsymbol{A}) \boldsymbol{E})+\nabla \times \boldsymbol{A}(\boldsymbol{r} \boldsymbol{E})+(\nabla \boldsymbol{A})(\boldsymbol{E} \times \boldsymbol{r})+\boldsymbol{A} \times \boldsymbol{E} \\
& +\boldsymbol{E} \times \boldsymbol{A}-(\boldsymbol{A} \times \operatorname{rot} \boldsymbol{E}) \times \boldsymbol{r}+(\boldsymbol{E} \times \boldsymbol{r}) \operatorname{div} \boldsymbol{A}-\boldsymbol{E}(\boldsymbol{A} \operatorname{rot} \boldsymbol{r})\} d V
\end{aligned}
$$

Die Ableitungen $\nabla$ wirken auf alles, was dahinter steht, also jeweils auf den ganzen Integranden. Entsprechend verschwinden diese Integrale wegen der Bedingungen im Unendlichen. Das vorletzte Glied verschwindet in Coulomb-Eichung, das davorstehende, wenn man unter $\boldsymbol{E}$ das Coulomb-Feld der Ladung versteht. Es bleibt nur das erste Glied übrig.

Der Ausdruck $\boldsymbol{r} \times \boldsymbol{e A}$ stellt also in Coulomb-Eichung den Felddrehimpuls (= Raumintegral über die Feld-Drehimpuls-Dichte) dar, der durch das Coulomb-Feld $\boldsymbol{E}^{i}$ der Ladung $e$ mit dem äußeren Magnetfeld $\boldsymbol{B}^{e}$ entsteht, und zwar auch dann, wenn die Ladung ruht. Ein weiterer Feld-Drehimpuls, der durch das von der mit der Geschwindigkeit $\boldsymbol{v}$ bewegten Ladung $e^{i}$ erzeugte Magnetfeld mit einem äußeren elektrischen Feld entsteht, ist hier nicht enthalten, sondern wird dem äußeren System zugeschlagen.

Auch diese Identifikation ist nicht neu, sondern in früheren Publikationen (P66, CT2) nachlesbar. 


\subsection{Magnetische Wechselwirkung}

$$
\begin{aligned}
e \boldsymbol{v} \boldsymbol{A} & =\int \rho \boldsymbol{v} \boldsymbol{A} d V=\int \boldsymbol{j} \boldsymbol{A} d V=\int \boldsymbol{A}^{e}\left(\operatorname{rot} \boldsymbol{B}^{i}-\frac{\partial \boldsymbol{E}^{i}}{\partial t}\right) d V \\
& =-\int \boldsymbol{A}^{e} \frac{\partial \boldsymbol{E}^{i}}{\partial t} d V+\int \nabla_{1}\left(\boldsymbol{B}_{1} \times \boldsymbol{A}_{2}\right) d V \\
& =\int \operatorname{div}(\boldsymbol{B} \times \boldsymbol{A}) d V-\int \boldsymbol{B}_{1}\left(\boldsymbol{A}_{2} \times \nabla_{2}\right) d V=+\int \boldsymbol{B}^{i} \boldsymbol{B}^{e} d V .
\end{aligned}
$$

Das Integral über $\boldsymbol{A} \partial \boldsymbol{E} / \partial t$ verschwindet, wenn man unter $\boldsymbol{E}$ das wirbelfreie Coulomb-Feld der Ladung versteht, da $\boldsymbol{A}$ in Coulomb-Eichung quellenfrei ist. Das Integral über die Divergenz ist (Gauß) ebenfalls Null.

Damit ist $e \boldsymbol{v} \boldsymbol{A}$ in dieser Eichung die magnetische Wechselwirkungsenergie aus dem Magnetfeld, welches die mit $\boldsymbol{v}$ bewegte Ladung $e$ erzeugt, und dem äußeren Magnetfeld, welches Anlaß ist für das Vektorpotential $\boldsymbol{A}$ am Ort der Ladung.

Chandrasekhar und Fermi (1953) führten die Energiedichte des Magnetfeldes ${ }^{1} / 2 \boldsymbol{B}^{2}$ in den Virialsatz für das interstellare Medium im wesentlichen ein elektrisch neutrales, aber stark ionisiertes Gas ein. Der Beweis in Teilchenformulierung unter Verwendung des Vektorpotentials wurde, soweit ich weiß, erstmalig von mir (P66) gegeben. In einem solchen Gas ist, wie schon in der Einleitung begründet, ein makroskopisches Magnetfeld (via gerichtete Ströme innerhalb des Gases) ein reines Wechselwirkungsfeld. Wegen der starken Ladungsneutralität ist das elektrische Wechselwirkungsfeld demgegenüber vernachlässigbar. Im interstellaren Gas ist die potentielle Energie (nahezu) vollständig gravitativen Ursprungs.

\section{Lagrange-Funktionen}

Für eine einzelne Ladung wird die Bewegungsgleichung (= Änderung der Geschwindigkeit bzw. des kinetischen Impulses) ebenso wie die Impulsgleichung (= Änderung des kanonischen Impulses) durch die bekannte Lagrange-Funktion

$$
\mathbf{L}_{0}(\boldsymbol{r}, \boldsymbol{v}, t)=\frac{1}{2} m \boldsymbol{v}^{2}+e \boldsymbol{v} \boldsymbol{A}(\boldsymbol{r}, t)-e U(\boldsymbol{r}, t)
$$

mit den unabhängigen Koordinaten Ort $\boldsymbol{q}(=\boldsymbol{r})$ und Geschwindigkeit $\boldsymbol{v}=d \boldsymbol{q} / d t$ sowie der Zeit $t$ und mit dem zur Ortskoordinate $\boldsymbol{q}$ kanonisch konjugierten Impuls 


$$
\boldsymbol{p}=\frac{\partial \mathbf{L}}{\partial \boldsymbol{v}}=m \boldsymbol{v}+e \boldsymbol{A}(\boldsymbol{r}, t)
$$

dargestellt. Sie enthält neben der kinetischen Energie und der (negativ gezählten) potentiellen Energie auch - in Coulomb-Eichung - die magnetische Wechselwirkungsenergie. $\mathbf{L}_{0}$ ist nur dann nicht explizit zeitabhängig, wenn es sich um eine Bewegung in zeitunabhängigen äußeren Feldern handelt. Die Impulsgleichung lautet

$$
\frac{d \boldsymbol{p}}{d t}=\frac{\partial \mathbf{L}}{\partial \boldsymbol{q}}=-\nabla(e U-e \boldsymbol{v} \boldsymbol{A}) .
$$

Die Form dieser Gleichung ist eichunabhängig, also sozusagen nicht ganz so „unphysikalisch“ wie der kanonische Impuls selbst. Bei einer Eichtransformation ändern sich zwar beide Seiten der Gleichung, jedoch in gleicher Weise.

An dieser Stelle ist eine Lehrbuch-Aussage (z.B. CT1) ergänzbar: „Obwohl sich die Lorentzkraft nicht aus einem Potential ableiten läßt, existiert eine Lagrange-Funktion".

Man kann sich ebenso gut folgerichtiger und vor allem positiver auf Newton - also nicht gerade neueste Literatur - beziehen, der die Kraft bekanntlich mit der Impulsänderung korrelierte:

Aus dem kinetischen Impuls $\boldsymbol{p}=m \boldsymbol{v}$ wird hier der kanonische oder generalisierte Impuls $\boldsymbol{p}^{*}=m \boldsymbol{v}+\boldsymbol{e A}$.

Aus dem kinetischen Potential $V=e U$ wird hier das generalisierte oder ,kanonische“ Potential $V^{*}=e(U-\boldsymbol{v} \boldsymbol{A})$.

Aus der kinetischen Potentialkraft $k=-\nabla V$ wird hier die generalisierte oder kanonische Potentialkraft $k^{*}=-\nabla V^{*}$.

Aus der kinetischen Bewegungsgleichung $d p / d t=-\nabla V$ wird hier die generalisierte, kanonische Bewegungsgleichung oder kanonische Impulsgleichung $\mathrm{d} p^{*} / \mathrm{d} t=-\nabla V^{*}$.

Mit der Interpretation des kanonischen Impulses als so etwas wie ein Gesamtimpuls einer Ladung kommt man wieder direkt auf Newton zurück:

Gesamtkraft = zeitliche Änderung des Gesamtimpulses.

Zur Erinnerung: $\mathbf{L}_{0}$ ist nicht eindeutig bestimmt. Fügt man die totale Zeitableitung einer beliebigen skalaren Funktion ef(r,t),

$$
\frac{d e f}{d t}=e \frac{\partial f}{\partial t}+e v \nabla f
$$

hinzu, so erhält man 


$$
\mathbf{L}_{0}^{\prime}=\frac{1}{2} m \boldsymbol{v}^{2}+e \boldsymbol{v}(\boldsymbol{A}+\nabla f)-e\left(U-\frac{\partial f}{\partial t}\right),
$$

Dies entspricht einer Eichtransformation, die ja bekanntlich weder an der Bewegungsgleichung noch - was weniger bekannt ist - an der Form der Impulsgleichung etwas ändert.

Für ein Vielteilchensystem kann man die Potentiale, wie oben erwähnt, als Superpositionen schreiben:

$$
\begin{aligned}
U_{i} & =\sum_{j} U_{i j}+U_{i e}\left(\boldsymbol{r}_{i}, t\right) \quad \text { und } \\
\boldsymbol{A}_{i} & =\sum_{j} \boldsymbol{A}_{i j}+\boldsymbol{A}_{i e}\left(\boldsymbol{r}_{i}, t\right)
\end{aligned}
$$

mit $j \neq i$. Für die Wechselwirkungspotentiale $U_{i j}$ und $\boldsymbol{A}_{i j}$ gibt es 2 Möglichkeiten: Erstens kann man sie als eine besondere Art äußerer Potentiale und damit als Funktionen von Ort der jeweiligen Ladung und Zeit ansehen: $U_{i j}\left(\boldsymbol{r}_{i}, t\right)$ und $\boldsymbol{A}_{i j}\left(\boldsymbol{r}_{i}, t\right)$. Da die wechselwirkenden Ladungen $j$ sich bewegen (ebenfalls Bewegungsgleichungen unterworfen sind), sind diese Potentiale notwendigerweise explizit zeitabhängig. Die dazugehörige Lagrange-Funktion ist gerade die Summe aller Einzelfunktionen:

$\mathbf{L}_{1}\left(\boldsymbol{r}_{i}, \boldsymbol{v}_{i}, \quad i=1, \ldots, N, t\right)=\sum_{i} \frac{1}{2} m_{i} \boldsymbol{v}_{i}^{2}+\sum_{i} e_{i} \boldsymbol{v}_{i} \boldsymbol{A}_{i}\left(\boldsymbol{r}_{i}, t\right)-\sum_{i} e_{i} U_{i}\left(\boldsymbol{r}_{i}, t\right)$.

Diese Lagrange-Funktion enthält offensichtlich die durch $U_{i j}$ und $\boldsymbol{A}_{i j}$ beschriebenen Wechselwirkungen zwischen zwei Ladungen $i$ und $j$ jeweils zweimal, nämlich für jede Ladung gänzlich, die Wechselwirkungen mit einem äußeren System $\left(U_{i e}\right.$ und $\left.\boldsymbol{A}_{i e}\right)$ dagegen nur einmal.

Ebenfalls zur Erinnerung der Beweis, dass diese $\boldsymbol{L}_{1}$ valide ist: Der kanonische Impuls folgt aus

$$
\boldsymbol{p}_{k}=\frac{\partial \mathbf{L}}{\partial \boldsymbol{v}_{k}}=m_{k} \boldsymbol{v}_{k}+e_{k} \boldsymbol{A}_{k}\left(\boldsymbol{r}_{k}, t\right)
$$

wie vorher. Die Impulsgleichung (mit $\partial / \partial \boldsymbol{q}_{k}=\nabla_{k}$ ) ist - ebenfalls wie vorher -

$$
\frac{d \boldsymbol{p}_{k}}{d t}=\frac{\partial \mathbf{L}}{\partial \boldsymbol{q}_{k}}=-e_{k} \nabla_{k}\left(U_{k}-\boldsymbol{v}_{k} \boldsymbol{A}_{k}\right)
$$

mit 


$$
\begin{aligned}
e_{k} \nabla_{k}\left(\boldsymbol{v}_{k} \boldsymbol{A}_{k}\right) & =e_{k} \boldsymbol{v}_{k} \times\left(\nabla_{k} \times \boldsymbol{A}_{k}\right)+e_{k}\left(\boldsymbol{v}_{k} \nabla_{k}\right) \boldsymbol{A}_{k} \\
& =e_{k} \boldsymbol{v}_{k} \times \boldsymbol{B}_{k}+e_{k} \frac{d \boldsymbol{A}_{k}}{d t}-e_{k} \frac{\partial \boldsymbol{A}_{k}}{\partial t},
\end{aligned}
$$

sodaß sich letzlich mit

$$
\boldsymbol{E}_{k}=-\nabla_{k} U_{k}-\frac{\partial \boldsymbol{A}_{k}}{\partial t}
$$

die jeweilige (kinetische) Bewegungsgleichung der Ladung

$$
\frac{d m_{k} \boldsymbol{v}_{k}}{d t}=\frac{d \boldsymbol{p}_{k}}{d t}-\frac{d e_{k} \boldsymbol{A}_{k}}{d t}=e_{k} \boldsymbol{E}_{k}+e_{k} \boldsymbol{v}_{k} \times \boldsymbol{B}_{k}
$$

ergibt. Diese Lagrange-Funktion ist zwar wohlbekannt, aber wird z.B. bei CT1 nie in voller Länge explizit hingeschrieben oder gar diskutiert, so daß unklar bleibt, von welchen Größen die Wechselwirkungspotentiale abhängen sollen. Die simple Tatsache, daß $\mathbf{L}_{1}$ nur dann eine valide Lagrange-Funktion ist, wenn diese als Funktionen von $\boldsymbol{r}_{i}$ und $t$ gegeben werden, habe ich nirgendwo erwähnt gefunden.

Da alle Summenterme in $\mathbf{L}_{1}$ jeweils nur von den Koordinaten eines einzigen Teilchens abhängen, kann man im Prinzip für jede Ladung eine eigene Eichung bzw. eine eigene Eichtransformation mit $f_{i}\left(\boldsymbol{r}_{i}, t\right)$ angeben. Eine generelle Umeichung mit einer für alle Ladungen gleichen Transformationsfunktion $f(\boldsymbol{r}, t)$ erfordert, sie für jede Ladung getrennt in $\mathbf{L}_{1}$ einzufügen:

$$
\mathbf{L}_{1}^{\prime}=\sum_{i}\left\{\frac{1}{2} m_{i} \boldsymbol{v}_{i}^{2}+e_{i} \boldsymbol{v}_{i}\left(\boldsymbol{A}_{i}+\nabla_{i} f\left(\boldsymbol{r}_{i}, t\right)\right)-e_{i}\left(U_{i}-\left.\frac{\partial f}{\partial t}\right|_{r=r_{i}}\right)\right\} .
$$

Wie oben erwähnt, ist diese Lagrange-Funktion notwendig explizit zeitabhängig, sobald die Wechselwirkung zwischen freien Ladungsträgern beschrieben werden soll, nicht nur der Einfluß konstanter externer Felder.

Will man nun diese Wechselwirkung genauer untersuchen, braucht man die Wechselwirkungspotentiale in expliziter Form. Dazu ist es freilich nötig, eine Näherung zu benützen. Will man die Einführung von neuen Variablen, nämlich Beschleunigungen, in die LagrangeFunktion vermeiden, so hat man sich auf eine quasistationäre Näherung zweiter Ordnung in $v / c$ zu beschränken (LL63). Dies ist auch die höchste Näherung, die keine Strahlungsdämpfung enthält, so dass man die Existenz einfacher Erhaltungssätze erwarten darf. Sie ist zudem keine unzulässig einschränkende Näherung, denn mit der Einführung 
der kinetischen Energie in die Lagrange-Funktion hat man sich ja bereits mit einer Näherung zweiter Ordnung in $v / c$ zufrieden gegeben.

Nach LL63 lauten die expliziten Potentiale in dieser Näherung und in Coulomb-Eichung (abgesehen von Maßsystemskonstanten)

$$
\begin{gathered}
U_{i j}=\frac{e_{j}}{r_{i j}}, \\
\boldsymbol{A}_{i j}=\frac{1}{2} e_{j}\left\{\frac{\boldsymbol{v}_{j}}{r_{i j}}+\frac{\boldsymbol{r}_{i j}\left(\boldsymbol{v}_{j} \boldsymbol{r}_{i j}\right)}{r_{i j}^{3}}\right\} .
\end{gathered}
$$

Insbesondere stimmt also in dieser Eichung das skalare Potential mit dem der nullten Näherung $(\boldsymbol{A}=0)$ überein. Wie die oben gegebene Deutung der Potentiale erfordert, sind die Wechselwirkungsenergien symmetrisch in den jeweils zwei beteiligten Ladungen $i$ und $j$,

$$
e_{i} U_{i j}=e_{j} U_{j i} \quad \text { und } \quad e_{i} \boldsymbol{v}_{i} \boldsymbol{A}_{i j}=e_{j} \boldsymbol{v}_{j} \boldsymbol{A}_{j i} .
$$

Möchte man eine (bis auf eine eventuelle Zeitabhängigkeit der äußeren Felder) nicht explizit zeitabhängige Lagrange-Funktion haben, so sind diese expliziten Formen zu benützen. Daß sich bei einem solchen Schritt auch die Lagrange-Funktion ändert, nicht nur die Darstellung der Potentiale, habe ich in der Literatur nicht erwähnt gefunden. Vielleicht sollte ich mich hier genauer ausdrücken: Daß sich bei dieser anderen Darstellung der Potentiale auch die Summierung ändert (wie gleich im nächsten Absatz beim skalaren Potential), ist durchaus bekannt, aber daß dies eine substantielle Änderung der LagrangeFunktion bedeutet, wird verschwiegen.

Aus der Mechanik ist die explizite Schreibweise für die skalare Wechselwirkung bzw. deren Energie und die Übertragung auf andere skalare Wechselwirkungen wohlbekannt. Für Ladungen werden sie als

$$
V=\frac{1}{2} \sum_{i} \sum_{j} e_{i} U_{i j}\left(r_{i j}\right)
$$

geschrieben. Hier geht $\Sigma_{i}$ über alle Ladungen, $\Sigma_{j}$ über alle mit $j \neq i$. Der Faktor $1 / 2$ deutet an, daß hier die Wechselwirkungsenergien zwischen zwei Teilchen $i$ und $j, e_{i} U_{i j}$ oder $e_{j} U_{j i}$, gemittelt jeweils zur Hälfte jedem Teilchen zugeschlagen werden. Eine andere Schreibweise wäre, den Faktor $1 / 2$ zu vermeiden, indem $\Sigma_{j}$ nur über $j<i$ erstreckt wird. Diese Schreibweise setzt die Symmetrie der expliziten Potentialenergien direkt voraus. Zwar ist eine solche Voraussetzung durch die obige 
Deutung der Terme notwendig, aber ohne diese Deutung ist es jedenfalls nicht unmittelbar einleuchtend, daß sie unvermeidbar wäre. ist

Die entsprechende, ebenfalls nicht unbekannte Lagrange-Funktion $\mathbf{L}_{2}=\sum_{i} \frac{1}{2} m_{i} \boldsymbol{v}_{i}^{2}+\sum_{i} e_{i} \boldsymbol{v}_{i} \boldsymbol{A}_{i}\left(\boldsymbol{r}_{i}, t\right)-\frac{1}{2} \sum_{i} \sum_{j} e_{i} U_{i j}\left(r_{i j}\right)-\sum_{i} U_{i e}\left(\boldsymbol{r}_{i}, t\right) \neq \mathbf{L}_{1}$.

Sie enthält, im Gegensatz zu $\mathbf{L}_{1}$, die Energie der skalaren Teilchenwechselwirkung nur einmal, die magnetische Teilchenwechselwirkung dagegen wie $\mathbf{L}_{1}$ zweimal. Umeichungen sind wie bei $\mathbf{L}_{1}$. Die Terme $\partial f / \partial t$ können bei $U_{e}$ angebracht werden, ohne daß die Wechselwirkungsterme verändert werden müssen. Hat man jedoch ein abgeschlossenes System ohne äußere Felder oder Potentiale, d.h. $U_{i e}=0$, so wäre es nicht sinnvoll, über eine Eichtransformation künstlich eine Art äußeres Potential einzuführen. Formal ist dies vermeidbar, solange $f$ zeitunabhängig ist.

Nicht aus der Literatur bekannt ist mir die von $\mathbf{L}_{2}$ verschiedene Form $\mathbf{L}_{3}$ der Lagrange-Funktion, in der auch der magnetische Anteil explizit gegeben ist. Der Wechselwirkungsanteil ist, wie oben begründet, ebenfalls mit dem Faktor $1 / 2$ zu versehen. Diese Lagrange-Funktion, eine konsequente Näherung zweiter Ordnung in $v / c$, lautet entsprechend

$$
\begin{aligned}
\mathbf{L}_{3}=\sum_{i} \frac{1}{2} m_{i} \boldsymbol{v}_{i}^{2}+\sum_{i} e_{i}\{ & \frac{1}{2} \sum_{j}\left(\boldsymbol{v}_{i} \boldsymbol{A}_{i j}\left(\boldsymbol{v}_{j}, \boldsymbol{r}_{i j}\right)-U_{i j}\left(r_{i j}\right)\right) \\
& \left.+\boldsymbol{v}_{i} \boldsymbol{A}_{i e}\left(\boldsymbol{r}_{i},(t)\right)-U_{i e}\left(\boldsymbol{r}_{i},(t)\right)\right\} .
\end{aligned}
$$

Sie ist nicht explizit zeitabhängig, solange es die äußeren Potentiale $\boldsymbol{A}_{i e}$ und $U_{i e}$ nicht sind.

Hier gilt nun als erstes zu zeigen, daß diese Lagrange-Funktion valide ist. Man findet für den kanonisch konjugierten Impuls

$$
\frac{\partial \mathbf{L}_{3}}{\partial \boldsymbol{v}_{k}}=m_{k} \boldsymbol{v}_{k}+\frac{1}{2} \sum_{j} e_{k} \boldsymbol{A}_{k j}+\frac{1}{2} \sum_{i} e_{i} \boldsymbol{v}_{i} \frac{\partial \boldsymbol{A}_{i k}}{\partial \boldsymbol{v}_{k}}+e_{k} \boldsymbol{A}_{k e}
$$

$(i \neq k$ und $j \neq k)$ mit

$$
e_{i} \boldsymbol{v}_{i} \frac{\partial \boldsymbol{A}_{i k}}{\partial \boldsymbol{v}_{k}}=\frac{\partial\left(e_{i} \boldsymbol{v}_{i} \boldsymbol{A}_{i k}\right)}{\partial \boldsymbol{v}_{k}}=\frac{\partial\left(e_{k} \boldsymbol{v}_{k} \boldsymbol{A}_{k i}\right)}{\partial \boldsymbol{v}_{k}}
$$

wegen der Symmetrie, daher 


$$
\boldsymbol{p}_{k}=m_{k} \boldsymbol{v}_{k}+e_{k} \boldsymbol{A}_{k}
$$

wie erwartet. Für $\partial \mathbf{L} / \partial \boldsymbol{r}_{k}$ ergibt sich, daß nur die Terme $\boldsymbol{A}_{k j}, \boldsymbol{A}_{i k}, \boldsymbol{A}_{k e}$, $U_{i k}, U_{k j}$ und $U_{k e}$ Beiträge liefern:

$$
\begin{aligned}
\frac{\partial \mathbf{L}}{\partial \boldsymbol{r}_{k}}=\nabla_{k}\{ & \frac{1}{2}\left(\sum_{i \neq k} e_{i}\left(\boldsymbol{v}_{i} \boldsymbol{A}_{i k}-U_{i k}\right)+\sum_{j \neq k} e_{k}\left(\boldsymbol{v}_{k} \boldsymbol{A}_{k j}-U_{k j}\right)\right) \\
& \left.+e_{k} \boldsymbol{v}_{k} \boldsymbol{A}_{k e}-e_{k} U_{k e}\right\} .
\end{aligned}
$$

Wieder führt die Symmetrie der Potentiale dazu, daß die beiden Summen mit dem Faktor $1 / 2$ gleich sind. Also ergibt sich

$$
\begin{aligned}
\frac{\partial \mathbf{L}}{\partial \boldsymbol{r}_{k}} & =\nabla_{k}\left(e_{k} \boldsymbol{v}_{k} \boldsymbol{A}_{k}-e_{k} U_{k}\right)=e_{k} \boldsymbol{v}_{k} \times\left(\operatorname{rot} \boldsymbol{A}_{k}\right)+e_{k}\left(\boldsymbol{v}_{k} \nabla_{k}\right) \boldsymbol{A}_{k}-e_{k} \operatorname{grad} U_{k} \\
& =e \boldsymbol{v} \times \boldsymbol{B}+\frac{d e \boldsymbol{A}}{d t}-e \frac{\partial \boldsymbol{A}}{\partial t}-\operatorname{grad}\left(e_{k} U_{k}\right)=e \boldsymbol{v} \times \boldsymbol{B}+e \boldsymbol{E}+e \frac{d \boldsymbol{A}}{d t}=\frac{d \boldsymbol{p}_{k}}{d t},
\end{aligned}
$$

d.h. die Impulsgleichung (und damit die Bewegungsgleichung) für die Ladung $\mathrm{k}$.

In dieser Version erscheinen, wie erwähnt, die Wechselwirkungsenergien zwischen zwei Teilchen jeweils nur einmal, in der obigen, explizit zeitabhängigen Form $\mathbf{L}_{1}$ dagegen zweimal. In Worten: $\mathbf{L}_{3}$ besteht aus der gesamten kinetischen Energie, der (negativ genommenen) gesamten potentiellen (= elektrischen) Wechselwirkungsenergie zwischen den Ladungen und mit einem äußeren Feld, und der gesamten magnetischen Wechselwirkungsenergie, ebenfalls zwischen den Ladungen und gegebenenfalls mit einem äußeren Magnetfeld.

In $\mathbf{L}_{3}$ lassen sich die einzelnen Wechselwirkungspotentiale direkt umeichen. Denn nur die explizite Form erlaubt es, die Koordinaten des Wechselwirkungspartners aktiv in die Eichung einzubeziehen.

Es sei noch einmal darauf hingewiesen, daß ein und dasselbe Problem mit substantiell verschiedenen Lagrange-Funktionen beschrieben werden kann. Es gibt aber nur eine, die, soweit es das Problem überhaupt erlaubt, nicht explizit zeitabhängig ist. Daß in ihr die Strahlungsdämpfung vernachlässigt werden muß, wurde bereits erwähnt.

\section{Hamilton-Funktionen $\mathbf{H}$}

Sie leiten sich aus den jeweiligen Lagrange-Funktionen mittels

$$
\mathbf{H}=\sum_{i} \boldsymbol{p}_{i} \boldsymbol{v}_{i}-\mathbf{L}
$$


ab. Die unabhängigen, kanonisch konjugierten Variablen sind $\boldsymbol{q}_{i}=\boldsymbol{r}_{i}$ und $\boldsymbol{p}_{i}=\partial \mathbf{L} / \partial \boldsymbol{v}_{i}$. Aus $\mathbf{L}_{1}$ ergibt sich bekanntermaßen

$$
\mathbf{H}_{1}=\sum_{i} \frac{1}{2 m_{i}}\left(\boldsymbol{p}_{i}-e_{i} \boldsymbol{A}_{i}\left(\boldsymbol{q}_{i}, t\right)\right)^{2}+\sum_{i} e_{i} U_{i}\left(\boldsymbol{q}_{i}, t\right) .
$$

Sie enthält, wie $\mathbf{L}_{1}$, die potentielle Energie der Wechselwirkung zwischen den Ladungen doppelt. Wie $\mathbf{L}_{1}$ ist sie notwendig explizit zeitabhängig, und zwar sogar auch in einer dem bekannten mechanischen Fall analogen statischen Näherung $\boldsymbol{A}=0$ (Näherung nullter Ordnung in $v / c$ ). Aus beiden Gründen ist es nicht empfehlenswert, hier von der Gesamtenergie $\mathrm{zu}$ sprechen, wie es fast üblich ist. Auch die zu $\mathbf{L}_{2}$ gehörige

$$
\mathbf{H}_{2}=\sum_{i} \frac{1}{2 m_{i}}\left(\boldsymbol{p}_{i}-e_{i} \boldsymbol{A}_{i}\left(\boldsymbol{q}_{i}, t\right)^{2}+\frac{1}{2} \sum_{i} \sum_{j} e_{i} U_{i j}\left(r_{i j}\right)+\sum_{i} e_{i} U_{i e}\left(\boldsymbol{q}_{i},(t)\right)\right.
$$

(mit $r_{i j}=\left|\boldsymbol{q}_{i}-\boldsymbol{q}_{j}\right|$ ) ist bekannt. Wie $\mathbf{H}_{1}$ ist sie über das Vektorpotential notwendig explizit zeitabhängig. Sie ist also, im Gegensatz zu $\mathbf{H}_{1}$, in nullter Ordnung in $v / c$, d.h. $\boldsymbol{A}=0$, zeitunabhängig und beschreibt dementsprechend die Erhaltungssätze des mechanischen Analogons. In zweiter Ordnung in $v / c$ ist sie explizit zeitabhängig und kann deshalb die Erhaltungssätze dieser Näherung nicht beschreiben.

Die Geschwindigkeit $\boldsymbol{v}_{i}$ einer Ladung $i$, die in der Lagrange-Formulierung eine der unabhängigen Variablen war, ist dies in einer Hamilton-Formulierung nicht mehr. Sie hängt sowohl in $\mathbf{H}_{1}$ als auch in $\mathbf{H}_{2}$ von beiden unabhängigen - den kanonischen - Variablen $\boldsymbol{q}_{i}$ und $\boldsymbol{p}_{i}$ der Ladung und außerdem explizit von der Zeit $t$ ab:

$$
\boldsymbol{v}_{i}=\frac{1}{m_{i}}\left(\boldsymbol{p}_{i}-e_{i} \boldsymbol{A}_{i}\left(\boldsymbol{q}_{i}, t\right)\right)=\boldsymbol{v}\left(\boldsymbol{q}_{i}, \boldsymbol{p}_{i}, t\right) .
$$

Nicht in der Literatur findet man die entweder direkt aus $\mathbf{L}_{3}$ abgeleitete oder über eine kanonische Transformation aus $\mathbf{H}_{1}$ oder $\mathbf{H}_{2}$ ableitbare $\mathbf{H}_{3}$, die ebenso wie $\mathbf{L}_{3}$ nur dann explizit zeitabhängig ist, wenn die äußeren Potentiale $U_{i e}\left(\boldsymbol{r}_{i},(t)\right)$ und $\boldsymbol{A}_{i e}\left(\boldsymbol{r}_{i},(t)\right)$ dies durch explizite Zeitabhängigkeit erzwingen. Sie enthält wie $\mathbf{L}_{3}$ auch das Vektorpotential der Wechselwirkungen $\boldsymbol{A}_{i j}$ in expliziter, nicht explizit zeitabhängiger Schreibweise und lautet

$$
\begin{aligned}
\mathbf{H}_{3}\left(\boldsymbol{q}_{i}, \boldsymbol{p}_{i}, \quad i=1, N\right)=\sum_{i} & \frac{1}{2} m_{i} \boldsymbol{v}_{i}^{2}+\frac{1}{2} \sum_{i} \sum_{j} e_{i} \boldsymbol{v}_{i} \boldsymbol{A}_{i j}\left(\boldsymbol{v}_{j}, \boldsymbol{r}_{i j}\right) \\
& +\frac{1}{2} \sum_{i} \sum_{j} e_{i} U_{i j}\left(r_{i j}\right)+\sum_{i} e_{i} U_{i e}\left(\boldsymbol{r}_{i},(t)\right)
\end{aligned}
$$


$\operatorname{mit} \boldsymbol{q}_{i}=\boldsymbol{r}_{i}, \boldsymbol{r}_{i j}=\boldsymbol{r}_{i}-\boldsymbol{r}_{j}, \boldsymbol{p}_{i}=m_{i} \boldsymbol{v}_{i}+e_{i} \boldsymbol{A}_{i}, \boldsymbol{A}_{i}=\sum_{j} \boldsymbol{A}_{i j}\left(\boldsymbol{v}_{j}, \boldsymbol{r}_{i j}\right)+\boldsymbol{A}_{i e}\left(\boldsymbol{r}_{i},(t)\right)$. Hier haben wir eine völlig neue Situation vor uns. Die Geschwindigkeit $\boldsymbol{v}_{i}$ einer Ladung $i$ ist über die Wechselwirkungspotentiale $\boldsymbol{A}_{i j}$ von allen anderen Geschwindigkeiten und Orten abhängig und damit eine (unbekannte) Funktion aller $2 \mathrm{~N}$ kanonisch konjugierten Variablen $\boldsymbol{q}$ und $\boldsymbol{p}$. Über das äußere Vektorpotential $\boldsymbol{A}_{i e}$ hängt sie zudem gegebenenfalls auch explizit von der Zeit ab. Wie üblich hängt die potentielle Wechselwirkung $U_{i j}$ nur vom Abstand $\left|\boldsymbol{q}_{i}-\boldsymbol{q}_{j}\right|=\left|\boldsymbol{r}_{i}-\boldsymbol{r}_{j}\right|=r_{i j}$ der Ladungen ab. $U_{i e}$ und $\boldsymbol{A}_{i e}$ hängen nur vom $\operatorname{Ort} \boldsymbol{q}_{i}=\boldsymbol{r}_{i}$ und gegebenenfalls explizit von der Zeit $t$ ab.

Der erste Term in $\mathbf{H}_{3}$ ist die kinetische Energie, der zweite die magnetische Wechselwirkung mit den anderen Ladungen, der dritte die entsprechende elektrische Wechselwirkung, und der letzte Term die elektrische Wechselwirkung mit einem (gegebenenfalls zeitabhängigen) vorgegebenen äußeren elektrischen Feld. Die in $\mathrm{L}_{3}$ auch enthaltene magnetische Wechselwirkung mit einem ebenfalls gegebenenfalls zeitabhängigen, aber vorgegebenen äußeren Magnetfeld fehlt wie in $\mathbf{H}_{2}$. Coulomb-Eichung ist bei dieser Deutung der einzelnen Terme wie früher vorausgesetzt. Im ersten Term ist bei $m_{i} \boldsymbol{v}_{i}=\boldsymbol{p}_{i}-e_{i} \boldsymbol{A}_{i}$ das ganze Vektorpotential $\boldsymbol{A}_{i}=\Sigma_{j} \boldsymbol{A}_{i j}+\boldsymbol{A}_{i e}\left(\boldsymbol{r}_{i},(t)\right)$ einzusetzen. Dieser Term (und damit jede einzelne Geschwindigkeit) ist also ebenso wie der zweite Term, aber im Gegensatz zum dritten, über das äußere Vektorpotential möglicherweise direkt explizit zeitabhängig.

Zunächst soll nachgewiesen werden, daß es sich hier um eine valide Hamilton-Funktion handelt. Die Ableitung von $\mathbf{H}_{3}$ aus $\mathbf{L}_{3}$ bzw. $\mathbf{H}_{1}$ oder $\mathbf{H}_{2}$ stellt das freilich schon sicher. Der direkte Nachweis besteht in der Berechnung von $\partial \mathbf{H}_{3} / \partial \boldsymbol{p}_{k}$ und $\partial \mathbf{H}_{3} / \partial \boldsymbol{q}_{k}$. Allgemein gilt

$$
\begin{gathered}
\frac{\partial}{\partial \boldsymbol{p}_{k}}=\sum_{i} \frac{\partial}{\partial \boldsymbol{v}_{i}} \frac{\partial \boldsymbol{v}_{i}}{\partial \boldsymbol{p}_{k}}, \\
\frac{\partial}{\partial \boldsymbol{q}_{k}}=\sum_{i} \frac{\partial}{\partial \boldsymbol{v}_{i}} \frac{\partial \boldsymbol{v}_{i}}{\partial \boldsymbol{q}_{k}}+\nabla_{k}
\end{gathered}
$$

mit unbekannten Ableitungen $\partial \boldsymbol{v} / \partial \boldsymbol{p}$ und $\partial \boldsymbol{v} / \partial \boldsymbol{q}$. Die gegebenenfalls nichtverschwindenden und ebenfalls unbekannten Ableitungen $\partial v / \partial t$ sind nicht von Belang.

Der erste Term von $\mathbf{H}_{3}$ ergibt (der obere Index c zeigt an, dass eine Größe bei der Differentiation konstant ist) 
$\frac{\partial \sum_{i} \frac{1}{2} m_{i} \boldsymbol{v}_{i}^{2}}{\partial \boldsymbol{p}_{k}}=\sum_{i} \frac{\partial \boldsymbol{v}_{i}^{c}\left(\boldsymbol{p}_{i}-\sum_{j} e_{i} \boldsymbol{A}_{i j}-e_{i} \boldsymbol{A}_{i e}\right)}{\partial \boldsymbol{p}_{k}}=\boldsymbol{v}_{k}-\sum_{i} \sum_{j} \frac{\partial\left(e_{i} \boldsymbol{v}_{i} \boldsymbol{A}_{i j}\right)}{\partial \boldsymbol{v}_{j}} \frac{\partial \boldsymbol{v}_{j}}{\partial \boldsymbol{p}_{k}}$

da $i \neq j$ und $\boldsymbol{A}_{i e}$ nur von $\boldsymbol{q}$ abhängt.

Beim zweiten Term ist die Symmetrie der Wechselwirkungsenergien wesentlich, d.h. man kann die Indizes nicht nur in einer Doppelsumme über alle Ladungen, sondern auch einzeln im Energieterm innerhalb der Summen vertauschen. Das Wechselwirkungspotential $\boldsymbol{A}_{i j}$ ist von $\boldsymbol{v}_{j}$ und vom nur $\boldsymbol{q}$-abhängigen Abstand $\boldsymbol{r}_{i j}=\boldsymbol{r}_{i}-\boldsymbol{r}_{j}$ abhängig. Das sollte übrigens sinnvollerweise nicht nur in der Coulomb-Eichung gelten. Für $\boldsymbol{e}_{i} \boldsymbol{v}_{i} \boldsymbol{A}_{i j}$ gilt so

$$
\frac{\partial}{\partial \boldsymbol{p}_{k}}=\frac{\partial}{\partial \boldsymbol{v}_{i}} \frac{\partial \boldsymbol{v}_{i}}{\partial \boldsymbol{p}_{k}}+\frac{\partial}{\partial \boldsymbol{v}_{j}} \frac{\partial \boldsymbol{v}_{j}}{\partial \boldsymbol{p}_{k}}
$$

also

$$
\begin{aligned}
\frac{1}{2} \sum \sum \frac{\partial\left(e_{i} \boldsymbol{v}_{i} \boldsymbol{A}_{i j}\right)}{\partial \boldsymbol{p}_{k}} & =\frac{1}{2} \sum \sum\left\{\frac{\partial\left(e_{i} \boldsymbol{v}_{i} \boldsymbol{A}_{i j}\right)}{\partial \boldsymbol{v}_{i}} \frac{\partial \boldsymbol{v}_{i}}{\partial \boldsymbol{p}_{k}}+\frac{\partial\left(\boldsymbol{e}_{i} \boldsymbol{v}_{i} \boldsymbol{A}_{i j}\right)}{\partial \boldsymbol{v}_{j}} \frac{\partial \boldsymbol{v}_{j}}{\partial \boldsymbol{p}_{k}}\right\} \\
& =+\sum \sum \frac{\partial\left(e_{i} \boldsymbol{v}_{i} \boldsymbol{A}_{i j}\right)}{\partial \boldsymbol{v}_{j}} \frac{\partial \boldsymbol{v}_{j}}{\partial \boldsymbol{p}_{k}} .
\end{aligned}
$$

Die beiden Terme der potentiellen Energie $U$ sind nur orts- (und gegebenenfalls zeit-) abhängig und geben deshalb keinen Beitrag. Es bleibt also

$$
\frac{\partial \mathbf{H}_{3}}{\partial \boldsymbol{p}_{k}}=\boldsymbol{v}_{k}=\frac{d \boldsymbol{q}_{k}}{d t}
$$

Die erste der Hamiltonschen Gleichungen ist erfüllt.

Bei der Berechnung von $\partial \mathbf{H}_{3} / \partial \boldsymbol{q}_{k}$ ist zunächst darauf hinzuweisen, $\mathrm{da} \partial \partial / \partial \boldsymbol{q}$ nur im Fall einer reinen Ortsfunktion mit dem einfachen Gradienten $\nabla$ übereinstimmt: Wie oben erwähnt, sind die Geschwindigkeiten $\boldsymbol{v}_{i}$ und $\boldsymbol{v}_{j}$ (in unbekannter Weise) Funktionen nicht nur von $\boldsymbol{p}$, sondern auch von $\boldsymbol{q}$, während immer $\nabla \boldsymbol{v}=0$. Daher gilt z.B. für $e_{i} \boldsymbol{v}_{i} \boldsymbol{A}_{i j}$ :

$$
\frac{\partial}{\partial \boldsymbol{q}_{k}}=\frac{\partial}{\partial \boldsymbol{v}_{i}} \frac{\partial \boldsymbol{v}_{i}}{\partial \boldsymbol{q}_{k}}+\frac{\partial}{\partial \boldsymbol{v}_{j}} \frac{\partial \boldsymbol{v}_{j}}{\partial \boldsymbol{q}_{k}}+\nabla_{k}
$$


Der erste Term führt auf

$$
\begin{aligned}
\sum_{i} \frac{\partial\left(\frac{1}{2} m_{i} \boldsymbol{v}_{i}^{2}\right)}{\partial \boldsymbol{v}_{i}} \frac{\partial \boldsymbol{v}_{i}}{\partial \boldsymbol{q}_{k}}= & \sum_{i} \boldsymbol{v}_{i} \frac{\partial m_{i} \boldsymbol{v}_{i}}{\partial \boldsymbol{q}_{k}}=\sum_{i} \boldsymbol{v}_{i} \frac{\partial \boldsymbol{p}_{i}}{\partial \boldsymbol{q}_{k}}-\sum_{i} \boldsymbol{v}_{i} \frac{\partial e_{i} \boldsymbol{A}_{i}}{\partial \boldsymbol{q}_{k}} \\
= & 0-\sum_{i} \sum_{j} \frac{\partial e_{i} \boldsymbol{v}_{i} \boldsymbol{A}_{i j}}{\partial \boldsymbol{v}_{j}} \frac{\partial \boldsymbol{v}_{j}}{\partial \boldsymbol{q}_{k}}-\sum_{i} \sum_{j} \nabla_{k} e_{i} \boldsymbol{v}_{i} \boldsymbol{A}_{i j} \\
& -\sum_{i} \nabla_{k} e_{i} \boldsymbol{v}_{i} \boldsymbol{A}_{i e}
\end{aligned}
$$

mit

$\sum \sum \nabla_{k} e_{i} \boldsymbol{v}_{i} \boldsymbol{A}_{i j}=\sum_{j} \nabla_{k} e_{k} \boldsymbol{v}_{k} \boldsymbol{A}_{k j}+\sum_{i} \nabla_{k} e_{i} \boldsymbol{v}_{i} \boldsymbol{A}_{i k}=2 \nabla_{k} \sum_{j} e_{k} \boldsymbol{v}_{k} \boldsymbol{A}_{k j}$

der zweite auf

$$
\begin{array}{r}
\frac{1}{2} \sum_{i} \sum_{j}\left(\frac{\partial e_{i} \boldsymbol{v}_{i} \boldsymbol{A}_{i j}}{\partial \boldsymbol{v}_{i}} \frac{\partial \boldsymbol{v}_{i}}{\partial \boldsymbol{q}_{k}}+\frac{\partial e_{i} \boldsymbol{v}_{i} \boldsymbol{A}_{i j}}{\partial \boldsymbol{v}_{j}} \frac{\partial \boldsymbol{v}_{j}}{\partial \boldsymbol{q}_{k}}+\nabla_{k} e_{i} \boldsymbol{v}_{i} \boldsymbol{A}_{i j}\right) \\
=+\sum \sum \frac{\partial e_{i} \boldsymbol{v}_{i} \boldsymbol{A}_{i j}}{\partial \boldsymbol{v}_{j}} \frac{\partial \boldsymbol{v}_{j}}{\partial \boldsymbol{q}_{k}}+\frac{1}{2} \sum \sum \nabla_{k} e_{i} \boldsymbol{v}_{i} \boldsymbol{A}_{i j}
\end{array}
$$

Der dritte und vierte Term führen in bekannter Weise auf

$$
\nabla_{k}\left(\frac{1}{2} \sum_{i} \sum_{j} e_{i} U_{i j}+\sum_{i} e_{i} U_{i e}\right)=\nabla_{k} e_{k} U_{k} .
$$

Zusammen ergibt sich so das richtige Ergebnis

$$
\frac{\partial \mathbf{H}_{3}}{\partial \boldsymbol{q}_{k}}=-\frac{d \boldsymbol{p}_{k}}{d t}=e_{k} \nabla_{k}\left(U_{k}-\boldsymbol{v}_{k} \boldsymbol{A}_{k}\right) .
$$

Damit ist auch die zweite Hamilton-Gleichung verifiziert.

Man braucht also die unbekannten Terme $\partial \boldsymbol{v} / \partial \boldsymbol{p}$ und $\partial \boldsymbol{v} / \partial \boldsymbol{q}$ nicht zu kennen, da sie sich gegenseitig herausheben, so daß nur bekannte Terme übrigbleiben.

Diese Hamilton-Funktion hat die unangenehme Eigenschaft, daß die Geschwindigkeiten nicht explizit als Funktion der kanonischen Koordinaten $\boldsymbol{q}, \boldsymbol{p}$ angegeben werden können, da jedes in $\boldsymbol{p}$ enthaltene Vektorpotential von allen anderen Geschwindigkeiten abhängt. Man begegnet hier also dem ungewöhnlichen Problem, dass diese Hamiltonfunktion überhaupt nicht explizit als Funktion ihrer unabhängigen Variablen $\boldsymbol{q}, \boldsymbol{p}$ angeschrieben werden kann. Aber sie ist die einzige 
Hamilton-Funktion, die auch in zweiter Näherung nicht explizit zeitabhängig ist, und sie ist die einzige, aus der die bekannten 7 skalaren Erhaltungskonstanten einer erhaltenden Wechselwirkung (Energie H, Impuls $\Sigma \boldsymbol{p}$, Drehimpuls $\Sigma \boldsymbol{q} \times \boldsymbol{p})$ ) direkt abgeleitet werden können.

Hier ist wieder einmal zu betonen, daß eine Hamilton-Funktion eben mehr darstellt, als nur eine andere Schreibweise der Bewegungsgleichung(en). Ebenso ist noch einmal darauf hinzuweisen, daß die Änderung des generalisierten Impulses sich aus einem generalisierten skalaren Potential ableiten läßt, ein in seiner Bedeutung vermutlich unterschätztes Analogon zur Mechanik.

\section{Erhaltungssätze}

$\mathbf{H}_{3}$ ist nicht explizit zeitabhängig außer über die externen Potentiale $U_{i e}, \boldsymbol{A}_{i e}$. Nur wenn diese zeitkonstant sind, wird die Energie in $\mathbf{H}_{3}$ erhalten. In einem abgeschlossenen System ohne externe Potentiale kann man also sinnvoll von Gesamtenergie sprechen. Das passt zu der hier verwendeten, schon in der Einleitung erwähnten Näherung, bei der Strahlungsdämpfung vernachlässigt wird. Ein klassisches System von Ladungsträgern kann ohne diese Vernachlässigung ja gar nicht abgeschlossen werden. Dieser Energiesatz ist mir nur aus makroskopischen Formulierungen (mit den entsprechenden Feld-Integralen statt den Potentialen) bekannt.

Der Grund dafür ist einfach: Bei der Wechselwirkung von einzelnen Ladungsträgern ist die elektrische Wechselwirkungsenergie klein gegen die Eigenenergie (Coulomb-Energie) der jeweiligen elektrischen Felder. Die elektrische Feldenergie besteht weitgehend aus den Eigenenergien. Diese Eigenenergien sind freilich in guter Näherung konstant und sollten deshalb eigentlich nicht in die Hamiltonfunktion, die Änderungen beschreiben soll, aufgenommen werden, ebenso wie trivialerweise die Ruheenergie $m c^{2}$ der Ladungen in $\mathbf{H}$ gewöhnlich nicht eingebracht wird. Es ist deshalb keine Fehler, nur inkonsequent, wenn - wie z.B. bei CT2 - die gesamte Feldenergie $\int^{1}{ }_{2} \boldsymbol{E}^{2} d V$ statt nur die Wechselwirkungsenergie in den Energieausdruck eingesetzt wird.

Es bedarf einer riesigen (makroskopischen) Anzahl von Ladungen, um die elektrische Wechselwirkungsenergie vergleichbar mit den Eigenenergien zu machen. Aber schon im Zweikörperproblem, z.B. dem (klassischen) Wasserstoffatom, ist die Wechselwirkungsenergie (potentielle Energie) von der gleichen Größenordnung wie die in der Hamilton-Funktion erscheinende kinetische Energie des Elektrons.

In ähnlicher Weise ist die magnetische Energie von höherer Ordnung in $v / c$ und damit gegenüber der skalar-potentiellen Energie gewöhnlich 
zunächst vernachlässigbar oder als Störung behandelbar. Makroskopisch kann man dagegen diese skalar-potentielle Energie durch Ladungs-Neutralität beliebig klein gegenüber der magnetischen Energie machen. Ich gab das Beispiel des interstellaren Mediums.

In einem System, das durch ein nicht explizit zeitabhängiges skalares Potential $V$ bestimmt ist, wird die Energie (kinetische plus potentielle) erhalten. Ein solches System heißt konservativ. Zumindest ursprünglich bezieht sich die Bezeichnung ,konservativ“ auf die Erhaltung der Energie. Insofern ist es also nicht richtig, den Fall der durch ein etwas komplizierteres, aber effektiv ebenfalls skalares Potential $e(U-\boldsymbol{v A})$ bestimmten elektromagnetischen Wechselwirkung, deren Energieerhaltung ja seit rund 150 Jahren unwidersprochen ist, als nicht konservativ zu bezeichnen oder gegen den konservativen Fall abzusetzen.

Für die Impulsbilanz benützt man das obige Ergebnis, daß $\partial \mathbf{H}_{3} / \partial \boldsymbol{q}_{k}$ als gewöhnlicher Gradient eines Energieausdrucks $e(U-\boldsymbol{v A})$ darstellbar ist. Beide Potentiale $U$ und $\boldsymbol{A}$ hängen bezüglich der Wechselwirkung $k j$ nur von den relativen Ortskoordinaten $\boldsymbol{r}_{k j}=\boldsymbol{r}_{k}-\boldsymbol{r}_{j}$ ab. Ihre Summierung hebt sich ebenso auf wie im üblicheren Fall eines „nur“ skalaren Potentials. Es bleibt

$$
\sum_{k} \frac{d \boldsymbol{p}_{k}}{d t}=-\sum_{k} e_{k} \frac{\partial\left(U_{k e}-\boldsymbol{v}_{k} \boldsymbol{A}_{k e}\right)}{\partial \boldsymbol{r}_{k} .}
$$

Wie zu erwarten, wird in einem abgeschlossenen System (ohne äußere Potentiale) der Gesamtimpuls, dargestellt durch den kanonisch konjugierten Impuls, erhalten - ein Ergebnis, das ebenso trivial ist wie es bisher in einer Teilchendarstellung noch nicht oder nicht allgemein verständlich formuliert wurde.

Mit diesem Ergebnis sieht man besonders leicht, wie eine weitere negative Lehrbuchaussage ins Positive verkehrt werden kann. Es heißt über das dritte Newtonsche Axiom actio= reactio (z.B. bei CT1): „Dieses Axiom gilt für Gravitationskräfte und elektrostatische Kräfte, jedoch nicht für magnetische Kräfte (deren Ursprung relativistischer Natur ist)". - Hier wird unter Kraft offensichtlich der Einfluß auf die Bewegung von Teilchen verstanden, also die Änderung des kinetischen Impulses. Die Felder dienen allein zur Vermittlung dieser Kräfte. Sieht man die Felder jedoch als essentiellen Bestandteil der Ladungen an, so ist der Gesamtimpuls zu betrachten, und dann gilt das Axiom eben auch im elektromagnetischen Fall. Dazu ist es freilich nötig, die Symmetrie der Wechselwirkungsenergien anzunehmen, was uns auf die quasistationäre Näherung zweiter Ordnung in $v / c$ für die Potentiale zurückführt, in der Strahlungsverluste vernachlässigt werden. Aber diese Näherung 
ist auch in dem in der Lehrbuchaussage angesprochenen elektrostatischen Fall $\boldsymbol{A}=0$ nötig, da ja die Probeladung, auf die eine Kraft wirkt, beschleunigt wird.

Ich formuliere deshalb neu: Dieses Axiom gilt für Gravitationskräfte in Newtonscher Näherung. Es gilt für elektrostatische Kräfte in nullter Näherung in $v / c$, d.h. Vernachlässigung der Bewegung aller Ladungen. Es gilt im allgemeineren elektrodynamischen Fall in zweiter Näherung in $v / c$, sofern man unter actio und reactio nicht den Einfluß auf die Bewegung, sondern im Newtonschen Sinne den Einfluß auf den Impuls versteht.

Wie auch im Fall eines rein skalaren Potentials, ist die Drehimpulsbilanz ein wenig komplizierter. Es gilt

$$
\boldsymbol{D}=\sum_{i} \boldsymbol{q}_{i} \times \boldsymbol{p}_{i}
$$

also

$$
\frac{d \boldsymbol{D}}{d t}=\sum_{i} \frac{d \boldsymbol{q}_{i}}{d t} \times \boldsymbol{p}_{i}+\sum_{i} \boldsymbol{q}_{i} \times \frac{d \boldsymbol{p}_{i}}{d t}=\sum_{i} \frac{d \mathbf{H}}{d \boldsymbol{p}_{i}} \times \boldsymbol{p}_{i}+\sum_{i} \frac{d \mathbf{H}}{d \boldsymbol{q}_{i}} \times \boldsymbol{q}_{i} .
$$

Von oben erhält man

$$
\begin{aligned}
\frac{d \boldsymbol{D}}{d t}=\sum_{i} \boldsymbol{v}_{i} \times \boldsymbol{p}_{i}+\sum_{i} e_{i} \nabla_{i}\left\{U_{i}-\boldsymbol{v}_{i} \boldsymbol{A}_{i}\right\} \times \boldsymbol{q}_{i} \\
=\sum_{i} e_{i}\left\{\boldsymbol{v}_{i} \times \sum_{j} \boldsymbol{A}_{i j}+\nabla_{i} \sum_{j}\left(U_{i j}-\boldsymbol{v}_{i} \boldsymbol{A}_{i j}\right) \times \boldsymbol{r}_{i}+\boldsymbol{v}_{i} \times \boldsymbol{A}_{i e}\right. \\
\left.+\nabla_{i}\left(U_{i e}-\boldsymbol{v}_{i} \boldsymbol{A}_{i e}\right) \times \boldsymbol{r}_{i}\right\} .
\end{aligned}
$$

Hier ist wieder $\boldsymbol{q}_{i}$ durch $\boldsymbol{r}_{i}$ ersetzt, weil jetzt nicht mehr die kanonische Variable $\boldsymbol{q}$ (von der auch $\boldsymbol{v}$ abhängen würde), sondern die gewöhnliche. Ortskoordinate $\boldsymbol{r}$ erscheint. Wie zu erwarten, heben sich die Wechselwirkungsglieder in jeder $i j$-Kombination paarweise gegeneinander auf:

$e_{i}\left(\boldsymbol{v}_{i} \times \boldsymbol{A}_{i j}+\nabla_{i}\left(U_{i j}-\boldsymbol{v}_{i} \boldsymbol{A}_{i j}\right) \times \boldsymbol{r}_{i}\right)+e_{j}\left(\boldsymbol{v}_{j} \times \boldsymbol{A}_{j i}+\nabla_{j}\left(U_{j i}-\boldsymbol{v}_{j} \boldsymbol{A}_{j i}\right) \times \boldsymbol{r}_{j}=0\right.$.

Diese Eigenschaft ist für das skalare Potential $U$ aus der Mechanik wohlbekannt. Zum Beweis benütze ich die expliziten Potentiale der Coulomb-Eichung. $U_{i j}=e_{j} u_{i j}$ ist nur vom Betrag $r_{i j}$ des Abstandes abhängig (hier $u_{i j}=1 / r_{i j}$ ). Wegen $\partial r_{i j} / \partial \boldsymbol{r}_{i}=-\partial r_{i j} / \partial \boldsymbol{r}_{j}=\boldsymbol{r}_{i j} / r_{i j}$ erhält man 


$$
e_{i} \nabla_{i} U_{i j} \times \boldsymbol{r}_{i}+e_{j} \nabla_{j} U_{j i} \times \boldsymbol{r}_{j}=-\frac{e_{i} e_{j}}{r_{i j}^{3}}\left(\boldsymbol{r}_{i j} \times \boldsymbol{r}_{i}-\boldsymbol{r}_{i j} \times \boldsymbol{r}_{j}\right)=0 .
$$

Für den ersten Teil des Vektorpotentials $\boldsymbol{A}_{i j}$ in Coulomb-Eichung, $1 / 2 e_{j} v_{j} / r_{i j}$, sieht man unmittelbar die Aufhebung der Terme, nämlich sowohl

$$
v_{i} \times v_{j}+v_{j} \times v_{i}=0
$$

als auch

$$
\left(\boldsymbol{v}_{i} \boldsymbol{v}_{j}\right)\left(\nabla_{i} \frac{1}{r_{i j}} \times \boldsymbol{r}_{i}+\nabla_{j} \frac{1}{r_{i j}} \times \boldsymbol{r}_{j}\right)=0 .
$$

Für den zweiten Teil des expliziten Vektorpotentials, ${ }_{1}^{1}{ }_{2} e_{j} \boldsymbol{r}_{i j}\left(\boldsymbol{v}_{j} \boldsymbol{r}_{j i}\right) / r_{\mathrm{ij}}^{3}$, gilt für die Ableitung von $1 / r^{3}$ dasselbe. Für die Zählerableitungen muß man dagegen beide Terme, die $\boldsymbol{A}$ enthalten, nämlich $\boldsymbol{v} \times \boldsymbol{A}$ und $\nabla(\boldsymbol{v} \boldsymbol{A})$, kombinieren. Der erste Term ergibt (ohne die Konstante $1 /{ }_{2} e_{i} e_{j} / r_{i j}^{3}$ )

$$
\left(\boldsymbol{v}_{i} \times \boldsymbol{r}_{i j}\right)\left(\boldsymbol{v}_{j} \boldsymbol{r}_{i j}\right)+\left(\boldsymbol{v}_{j} \times \boldsymbol{r}_{j i}\right)\left(\boldsymbol{v}_{i} \boldsymbol{r}_{j i}\right),
$$

die Zählerableitungen des zweiten mit $\nabla_{i}\left(\boldsymbol{v}_{i} \boldsymbol{r}_{i j}\right)=\boldsymbol{v}_{i}, \nabla_{j}\left(\boldsymbol{v}_{i} \boldsymbol{r}_{i j}\right)=-\boldsymbol{v}_{i}$, etc.,

$$
\begin{aligned}
& \left\{\left(\boldsymbol{v}_{j} \boldsymbol{r}_{i j}\right) \nabla_{i}\left(\boldsymbol{v}_{i} \boldsymbol{r}_{i j}\right)+\left(\boldsymbol{v}_{i} \boldsymbol{r}_{i j}\right) \nabla_{i}\left(\boldsymbol{v}_{j} \boldsymbol{r}_{i j}\right)\right\} \times \boldsymbol{r}_{i} \\
& +\left\{\left(\boldsymbol{v}_{i} \boldsymbol{r}_{j i}\right) \nabla_{j}\left(\boldsymbol{v}_{j} \boldsymbol{r}_{j i}\right)+\left(\boldsymbol{v}_{j} \boldsymbol{r}_{j i}\right) \nabla_{j}\left(\boldsymbol{v}_{i} \boldsymbol{r}_{j i}\right)\right\} \times \boldsymbol{r}_{j}=\left(\boldsymbol{v}_{j} \boldsymbol{r}_{i j}\right)\left(\boldsymbol{v}_{i} \times \boldsymbol{r}_{i}\right) \\
& +\left(\boldsymbol{v}_{i} \boldsymbol{r}_{i j}\right)\left(\boldsymbol{v}_{j} \times \boldsymbol{r}_{i}\right)+\left(v_{i} \boldsymbol{r}_{j i}\right)\left(\boldsymbol{v}_{j} \times \boldsymbol{r}_{j}\right)+\left(\boldsymbol{v}_{j} \boldsymbol{r}_{j i}\right)\left(\boldsymbol{v}_{i} \times \boldsymbol{r}_{j}\right)=\left(\boldsymbol{v}_{j} \boldsymbol{r}_{i j}\right)\left(\boldsymbol{v}_{i} \times \boldsymbol{r}_{i j}\right) \\
& +\left(\boldsymbol{v}_{i} \boldsymbol{r}_{i j}\right)\left(\boldsymbol{v}_{j} \times \boldsymbol{r}_{i j}\right),
\end{aligned}
$$

was, weil in der Bilanz negativ erscheinend, sich gegen den obigen ersten Term weghebt. Damit ist gezeigt, daß ein abgeschlossenes System von wechselwirkenden Ladungen den üblichen 7 skalaren Erhaltungssätzen folgt.

\section{Eichtransformationen}

Die obigen Ergebnisse gelten so, wie sie abgeleitet wurden, nur in der Coulomb-Eichung - man kann auch sagen, nur für den quellenfreien Teil des Vektorpotentials. Man kann diesem jedoch einen wirbelfreien Teil, d.h. den Gradienten eines Skalars, hinzufügen (gegebenenfalls muß dann auch das skalare Potential entsprechend ergänzt werden), ohne daß sich die Kräfte auf die Ladungen, d.h. elektrisches und Magnetfeld, ändern. Im Prinzip kann man also dem Vektorpotential 
an einem Ort und zu einer Zeit jeden beliebigen Wert zuordnen, ohne wie man sagt - an der Physik etwas zu ändern.

Ich fasse noch einmal zusammen: Das Vektorpotential $\boldsymbol{A}$ bzw. das Produkt $\boldsymbol{e A}$ erscheint im zum Ort kanonisch konjugierten Impuls, mit der Dimension eines Impulses. Also ist die Frage naheliegend, um was für einen Impuls es sich handelt. Der eichinvariante quellenfreie Teil des Vektorpotentials (bzw. eA) kann in der Tat mit einem für das physikalische Problem wesentlichen Impuls identifiziert werden. Der nichtinvariante wirbelfreie Teil, der sinnvollerweise ebenfalls die Dimension eines Impulses (bzw. Impulses pro Einheitsladung) haben sollte, ist dagegen nicht gedeutet (die Grüblsche (l.c.) willkürliche Eichkonvention).

Es gibt aber eine Gruppe von Eichtransformationen, für die eine physikalische Interpretation dieses nichtinvarianten Teils einfach möglich ist - sozusagen euphysikalische Transformationen. Sie vermitteln jeweils den Übergang zwischen zwei verschiedenen physikalischen Situationen, die nur lokal in den Feldern übereinstimmen. Hier ist dann also auch die Physik nicht eichinvariant.

Zunächst ist darauf hinzuweisen, daß der eichinvariante quellenfreie Teil von $\boldsymbol{A}$ über den gesamten Raum definiert sein sollte, um die Raumintegrale ausführen zu können. Eine Eichtransformation muß aber nur in dem Teil des Raumes wirbelfrei sein, in dem sich Ladungen befinden, auf die die Felder einwirken könnten. Ich gebe dazu ein bekanntes Beispiel:

Eine Ladung bewege sich geradlinig im feldfreien Raum, z.B. parallel zur $y$-Achse $(x=$ const $\neq 0, z=0)$. Das ist durch $\boldsymbol{A}=0$ beschreibbar. Das Skalarfeld für die Eichtransformation sei $f=\arcsin (x / r)$ mit $r^{2}=x^{2}+y^{2}$. Dessen Gradient (also $A$ ) besteht aus Kreisen um die $z$ Achse mit $A_{r}=0, A_{z}=0, A_{\varphi}=\mathrm{const} / r$. Dieses Vektorpotential entspricht einem Magnetfeldbündel entlang der $z$-Achse, wie es durch eine stromdurchflossene Spule entlang der $z$-Achse erzeugt wird. Es ist im interessanten Bereich, nämlich dort, wo sich die Ladung bewegt, wirbelfrei, also entsteht dort kein Kraftfeld. Es ist dort übrigens außerdem auch quellenfrei, trägt also direkt, ohne die Notwendigkeit einer Umeichung, zum Feldimpuls und zur magnetischen Feldenergie bei - auch wenn die Reaktion der Spule auf das Coulomb-Feld der vorbeifliegenden Ladung das Eindringen dieses Feldes in die Spule verhindert.

Um es kurz zu sagen: Der Aharanov-Bohm-Effekt entbehrt durchaus nicht klassischer Grundlagen. Aber - anschaulich gesprochen - geht das klassische Elektron, wenn auch nicht sein Feld, links oder rechts vorbei, während die Elektronenwelle durch die Spule hindurch läuft. 
Die Eichtransformationen der Potentiale sind zurückführbar auf Umschreibungen der Bewegungsgleichungen von Partikeln. Ich gehe aus von der Bewegung eines Teilchens $i$, die durch Ort $\boldsymbol{r}_{i}(t)$ und Geschwindigkeit $\boldsymbol{v}_{i}(t)=d \boldsymbol{r}_{i} / d t$ des Teilchens gekennzeichnet ist. Sie wird durch die Newtonsche Impulsgleichung bestimmt: Kraft $k=$ zeitliche Änderung des Impulses, für den ich, obwohl ursprünglich der kinetische Impuls $m v$ gemeint war, gleich die allgemeinere, kanonische Schreibung $\boldsymbol{p}$ einsetze. Die Kraft leite sich aus einem (skalaren) Potential $V$ ab:

$$
\boldsymbol{k}\left(\boldsymbol{r}_{i}, t\right)=-\operatorname{grad}_{i} V(\boldsymbol{r}, t) .
$$

Der Gradient wird am Ort $\boldsymbol{r}_{i}$ des Teilchens benötigt, das Potential muß also zunächst auch nur an dieser Stelle und sogar nur mit der ersten Ableitung bekannt sein. Eine entsprechende Potentialdefinition ist wegen der Zeitabhängigkeit - lokal immer möglich (wenn auch nicht immer praktikabel, z.B. im Fall einer magnetischen Kraft, wenn man unter $\boldsymbol{p}$ den kinetischen Impuls versteht). Für die Änderung des kanonischen Impulses im elektromagnetischen Fall gibt es jedoch das verallgemeinerte skalare Potential $V=e U-e v A$. Lokal ist also die Gleichung nicht mehr als eine stets erfüllte Identität.

Um zu einer physikalischen Aussage zu gelangen, um also die Bewegung zeitlich und örtlich verfolgen zu können, muß das Potential nicht nur lokal, sondern - ,quasilokal“ - in einer örtlichen und zeitlichen Umgebung von $\boldsymbol{r}_{i}$ und $t$ bekannt sein.

Die Newtonsche Impulsgleichung lautet dann

$$
\frac{d \boldsymbol{p}}{d t}=-\operatorname{grad} V
$$

Sie gilt nicht nur im mechanischen Fall, sondern unter Verallgemeinerung des Impulses und des Potentials ebenso im elektrodynamischen Fall. An dieser Gleichung ändert sich nichts, wenn man eine (mathematische) Identität $\boldsymbol{F}=\boldsymbol{F}$ addiert:

$$
\frac{d \boldsymbol{p}}{d t}+\boldsymbol{F}=-\operatorname{grad} V+\boldsymbol{F} .
$$

Sinnvollerweise muß $\boldsymbol{F}$ ein Vektor sein, die richtige Dimension haben und zur Zeit $t$ am Ort $\boldsymbol{r}_{i}$ bekannt sein. Die Impulsgleichung behält ihre Eigenschaft, für eine physikalische Interpretation zumindest quasilokal definiert zu sein, nur, wenn dies auch für $\boldsymbol{F}$ gilt. Dann ist $\boldsymbol{F}(\boldsymbol{r}, t)$ ein zumindest quasilokal definiertes Vektorfeld. Falls es Wirbel enthält, können diese entfernt werden, denn die rechte Seite der 
Impulsgleichung sollte wirbelfrei bleiben. Man kann also o.B.d.A. davon ausgehen, dass $\boldsymbol{F}$ wirbelfrei ist und sich als Gradient darstellen lässt. Dann lässt sich eine skalare Funktion $f(\boldsymbol{r}, t)$ finden, für die

$$
\nabla f=\int \boldsymbol{F} d t
$$

gilt. Damit ist o.B.d.A. eine beliebige Vektorfunktion $\boldsymbol{F}$ reduzierbar auf eine Skalarfunktion $f$. Wir sind zurück bei den Eichtransformationen. Aber auch das Umgekehrte gilt: Jede beliebige Umeichungsfunktion $f$ lässt sich in eine additive Vektorfunktion $\boldsymbol{F}$ umformen.

Die erweiterte Impulsgleichung behält o.B.d.A. die Form einer Newtonschen Impulsgleichung, wenn sich $\boldsymbol{F}$ lokal sowohl als totale Zeitableitung als auch als Gradient darstellen lässt, sich also aus dem lokalen Gradienten einer im Übrigen beliebigen, quasilokal bekannten Skalarfunktion $f$ ableitet:

$$
\boldsymbol{F}=\frac{d \nabla f}{d t}=\frac{\nabla d f}{d t}=\frac{\partial \nabla f}{\partial t}+(\boldsymbol{v} \nabla) \nabla f=\frac{\nabla \partial f}{\partial t}+\nabla(\boldsymbol{v} \nabla f) .
$$

Man erhält nun mit $\pi=\nabla f$

$$
\frac{d(\boldsymbol{p}+\pi)}{d t}=-\operatorname{grad}\left(V-\frac{\partial f}{\partial t}-\boldsymbol{v} \pi\right)
$$

eine Gleichung, die sofort als Eichtransformationsgleichung erkennbar ist. Damit kann also jede Eichtransformation der Potentiale als eine simple Addition zur Impulsgleichung verstanden werden.

Die Addition einer mathematischen Identität ist physikalisch nicht relevant. Hier ist entsprechend umgekehrt zu fragen, wie in der obigen Gleichung eine solche willkürliche Addition erkannt und rückgängig gemacht werden kann, um nichtrelevantes Beiwerk aus der Impulsgleichung zu entfernen.

Im mechanischen Fall ist dies einfach. Man kennt den Impuls $m v$. Das Vektorpotential sollte per definitionem Null sein. Jedes in der Impulsgleichung erscheinende wirbelfreie Vektorpotential $\pi$ kann lokal oder global durch eine Rücktransformation entfernt werden. Es gibt also eine eindeutige Eichung $\pi=0$. Als Eichunsicherheit bleibt nur eine in der Einleitung erwähnte zeitabhängige, aber ortsunabhängige Funktion $f(t)$.

Im elektrodynamischen Fall ist die Situation etwas komplizierter. Man weiß zwar, dass nur der wirbelhafte oder transversale Teil des Vektorpotentials physikalisch wirksam ist. Jedes global gegebene Vektorfeld lässt sich bis auf eine Konstante eindeutig in ein Wirbelfeld 
und eine Quellenfeld zerlegen. Das letztere lässt sich vollständig wegtransformieren (Coulomb-Eichung). Global ist daher die Unterscheidung zwischen physikalisch wirksamem und unwirksamem Vektorpotentialfeld eindeutig durchführbar. Darauf weist auch CT2 hin, wie oben erwähnt. $\mathrm{Ob}$ diese Eichung aus anderen Gründen, z.B. einer Lorentz-invarianten Formulierung, dennoch nicht sinnvoll erscheint, ist hier (noch) nicht relevant.

In einem beschränkten Gebiet ist eine Coulomb-Eichung jedoch nicht eindeutig möglich. Hier kann jedes Vektorfeld in drei (nicht eindeutige) Teile zerlegt werden: Ein Quellenfeld, ein Wirbelfeld, und ein quellen- und wirbelfreies ,Zwischenfeld“, dessen Zugehörigkeit zu der einen oder anderen Seite unbestimmbar ist.

Soweit ich sehe, liegt das Problem der Eichtransformationen genau an dieser Stelle. Ergänzt man das Zwischenfeld global durch ein Quellenfeld, so ist es physikalisch irrelevant und kann durch eine Eichtransformation entfernt oder modifiziert werden. Ergänzt man dasselbe Zwischenfeld global durch ein Wirbelfeld, d.h. durch Magnetfelder außerhalb des beschränkten Gebietes, so ist das Feld zumindest teilweise - nicht durch Eichtransformation entfernbar und so physikalisch relevant. Die Relevanz oder Irrelevanz dieses Vektorpotentials in einem beschränkten Gebiet kann nur durch Kenntnis der physikalischen Bedingungen im äußeren Gebiet festgestellt werden. Der Befund kann als plausible Folge des klassischen Dualismus Teilchen (lokalisiert) und Feld (ausgedehnt) - verstanden werden.

Dasselbe Problem taucht übrigens schon in der Mechanik und Elektrostatik mit der oben erwähnten ortsunabhängigen Funktion $f$ $(t)$ auf. Im Inneren einer Massenschale ist das Gravitationspotential konstant, im Inneren einer geladenen Kugel das skalare elektrostatische Potential; beides kann zeitabhängig sein. Welcher Teil dieses „hemiphysikalischen“ Potentials euphysikalisch äußere Bedingungen widerspiegelt oder aphysikalisch einer irrelevanten Eichtransformation nach obigem Muster entspringt, ist lokal nicht feststellbar.

An dieser Stelle wird es vielleicht besonders klar, dass das Eichproblem keine Frage der Elektrodynamik allein oder des Vektorpotentials ist. Es ist vielmehr in Bezug auf die Darstellung in Potenzen von $v / c$ ein Problem nullter Ordnung.

\section{Ausblick}

Der klassische elektrische Teilchen/Feld-Dualismus ist wenig diskutiert worden. Er kann aber zum Verständnis einfacher Interaktionen 
beitragen. $\mathrm{Da}$ z.B. ein abgeschlossenes System von Ladungsträgern Erhaltungssätzen genügen muß, ist zweifellos unwidersprochen, und das immerhin seit Julius Robert von Mayer. Diese Erhaltungssätze lassen sich im Teilchenbild (z.B. Hamilton-Funktion) darstellen, wobei Potentiale eine Rolle spielen. Daß auch die Felder zu den Erhaltungsgrößen beitragen, ist wohl seit James Clerk Maxwell ebenso klar. Insofern muß es notwendigerweise eine Beziehung zwischen Feldern und Potentialen geben. Auch daß es einer Näherung ohne Strahlung bedarf, um ein System von Ladungsträgern überhaupt abschließen zu können, ist selbstverständlich.

Die Deutung der Potentiale führt zu einem besseren Verständnis und zu einer bisher offenbar noch nicht formulierten Lagrange- bzw. Hamiltonfunktion, einschließlich der darin steckenden Erhaltungssätze, die bisher fehlten.

Auf zwei Aussagen weise ich besonders hin, weil sie meiner Meinung nach bisher nicht die nötige Beachtung gefunden haben. Die erste ist, dass die Form der Newtonschen Impulsgleichung nicht von der Eichung abhängt. Die Gleichung enthält zwar - ich zitiere wieder Grübl (l.c.) - neben physikalischen Fakten auch willkürliche Eichkonvention, aber dieselbe auf beiden Seiten der Gleichung. Die zweite ist, dass jede Umeichung sich als Addition einer mathematischen Identität interpretieren lässt. Diese ist bei globaler Kenntnis der physikalischen Gegebenheiten eindeutig von der physikalischen Aussage trennbar.

Aus der Mechanik weiß man, das ein abgeschlossenes wechselwirkendes System von Teilchen genau 7 skalare Erhaltungskonstanten besitzt, nämlich Energie, Impuls und Drehimpuls. In vielen mechanischen Problemen ist die Impuls- und Drehimpulsbilanz mehr oder weniger simpel, so daß das physikalische Problem sich gewissermaßen auf die Energiebilanz reduziert. Als Beispiel nenne ich nur aus der statistischen Mechanik die (groß)kanonische Gesamtheit - die viel überzeugender wäre, wenn Impuls und Drehimpuls wenigstens erwähnt würden. Denn daß ein Minimum der Energie $\mathbf{H}$ stets mit verschwindendem kinetischem (Gesamt)-Impuls und -Drehimpuls einhergeht, gilt nur in der Mechanik.

Im elektrodynamischen Fall gibt es dieselben Erhaltungskonstanten. Ihre Interpretation ist aber ein wenig schwieriger. Eine ruhende Ladung kann Impuls und Drehimpuls haben. Eine bewegte Ladung wiederum kann einen verschwindenden Impuls oder Drehimpuls haben. Als ein interessantes Beispiel führe ich hier nur die Londonsche Gleichung der Supraleitung an. Die aus dem Bohr-Lorentz-Van Leeuwenschen Theorem gezogenen Schlüsse sind für die Mechanik brauchbar, nicht für die Elektrodynamik. Das hat übrigens - um auf die Vorbemerkung zu 
dieser Arbeit zurückzukommen - schon Schrödinger als interessante Möglichkeit angesehen.

\section{Danksagung}

G. GRÜBL, Innsbruck, trug mit wertvollen Hinweisen zur Formulierung bei. Mircea Pfleiderer hat durch zahlreiche Fragen in die Klarstellung nicht nur des Textes, sondern insbesondere auch meiner Gedanken wesentlich eingegriffen.

\section{Literatur}

Chandrasekhar, S., Fermi, E. (1953) Astrophys. J. 118: 116

CT1 = COHEN-TANNOUdJI, C., DiU, B., LALOE, F. (1977 und 1986) Quantenmechanik I, 4. Aufl. 2009, II, 3. Aufl. 2008, de Gruyter (als Beispiel eines modernen Lehrbuchs) CT2 = Cohen-TAnNoudi, C., Dupont-Roc, J., Grynberg, G. (1997) Photons and Atoms: Introduction to Quantum Electrodynamics. Wiley Professional.

Grübl G. (2010) pers. Mitt.

LL63 = LANDAU, L. D., LIFSCHIZ, E. M. (1963) Lehrbuch der theoretischen Physik Band II, Feldtheorie, Akademie-Verlag, Berlin

v.LAUE, M. (1956) Die Relativitätstheorie II, Vieweg (Braunschweig), S. 186

MIE, G. (1912) Ann. Phys. 37: 511

P66 = PFLEIDERER, J. (1966) Zur klassischen elektromagnetischen Wechselwirkung von Punktladungen unter Berücksichtigung von Gliedern bis zur zweiten Ordnung in v/c, Forsch. Ber. Astron. Inst. Bonn 66-13

RoHrLICH, F. (1964) Classical charged particles. Neuauflage 2007: World Scientific

Author's address: Prof. Dr. Jörg Pfleiderer, Institut für Astro- und Teilchenphysik, Leopold-Franzens-Universität Innsbruck, Technikerstraße 25, 6020 Innsbruck, Österreich. E-Mail: joerg.pfleiderer@uibk.ac.at 